\title{
Microbunching instability in a chicane: Two-dimensional mean field treatment
}

\author{
Gabriele Bassi* \\ Department of Physics, University of Liverpool, Liverpool, L69 7ZE, United Kingdom, \\ and The Cockcroft Institute, Daresbury, WA4 4AD, United Kingdom \\ James A. Ellison ${ }^{\dagger}$ and Klaus Heinemann ${ }^{\ddagger}$ \\ Department of Mathematics and Statistics, University of New Mexico, Albuquerque, New Mexico 87131, USA \\ Robert Warnock ${ }^{\S}$ \\ SLAC National Accelerator Laboratory, Stanford University, Menlo Park, California 94025, USA, \\ and Lawrence Berkeley National Laboratory, University of California, Berkeley, California 94720, USA \\ (Received 1 December 2008; published 20 August 2009)
}

\begin{abstract}
We study the microbunching instability in a bunch compressor by a parallel code with some improved numerical algorithms. The two-dimensional charge/current distribution is represented by a Fourier series, with coefficients determined through Monte Carlo sampling over an ensemble of tracked points. This gives a globally smooth distribution with low noise. The field equations are solved accurately in the lab frame using retarded potentials and a novel choice of integration variables that eliminates singularities. We apply the scheme with parameters for the first bunch compressor system of FERMI@Elettra, with emphasis on the amplification of a perturbation at a particular wavelength and the associated longitudinal bunch spectrum. Gain curves are in rough agreement with those of the linearized Vlasov system at intermediate wavelengths, but show some deviation at the smallest wavelengths treated and show the breakdown of a coasting beam assumption at long wavelengths. The linearized Vlasov system is discussed in some detail. A new 2D integral equation is derived which reduces to a well-known 1D integral equation in the coasting beam case.
\end{abstract}

DOI: 10.1103/PhysRevSTAB.12.080704

PACS numbers: 29.27.Bd, 41.60.Ap, 41.60.Cr, 52.65.Ff

\section{INTRODUCTION}

Bunch compressors, designed to increase the peak current, can lead to a microbunching instability with detrimental effects on the beam quality. This is a major concern for free electron lasers (FELs) where very bright electron beams are required, i.e., beams with low emittance and energy spread [1-11]. In this paper we compare two models, i.e., we apply our self-consistent, parallel solver to study the microbunching instability in the first bunch compressor system of FERMI@Elettra and compare it with the coasting beam approximation in $[12,13]$. This bunch compressor system was proposed as a benchmark for testing codes at the September 2007 microbunching instability workshop in Trieste [14].

A basic theoretical framework for understanding this instability is the 3D Vlasov-Maxwell system (on 6D phase space). However, the numerical integration of this system is computationally too intensive at the moment. Our basic Ansatz is a 2D Vlasov-Maxwell system. More precisely, we treat the beam evolution through a bunch compressor using our Monte Carlo mean field self-consistent approxi-

\footnotetext{
*g.bassi@dl.ac.uk

†ellison@math.unm.edu

theineman@math.unm.edu

§warnock@slac.stanford.edu
}

mation. We randomly generate $\mathcal{N}$ points from an initial phase space density. Here we use $\mathcal{N}$ for the simulated points to distinguish it from $N$ for the number of particles in the beam. We then calculate the charge density using a smooth density estimation based on Fourier series. The electric and magnetic fields are calculated from the smooth charge/current density using a novel field formula that avoids singularities by using the retarded time as a variable of integration. The points are then moved forward in small time steps using the equations of motion in the beam frame with the fields frozen during a time step. We try to choose $\mathcal{N}$ large enough so that the charge density is a good approximation to the charge density that would be obtained from solving the 2D Vlasov-Maxwell system exactly. We call this method the Monte Carlo particle (MCP) method and the underlying model the MCP model. We believe that we calculate the charge density accurately and that for $\mathcal{N}$ sufficiently large one could obtain an accurate approximation to the Vlasov phase space density. That is beyond our current computer capability, however, and it is likely that a better approach would be to use the method of local characteristics to integrate the Vlasov equation directly.

Our MCP solver has been tested against other codes on the Zeuthen benchmark bunch compressors. Our results for the mean energy loss are in good agreement with $2 \mathrm{D}$ and $3 \mathrm{D}$ codes confirming that $1 \mathrm{D}$ codes underestimate the effect of coherent synchrotron radiation (CSR) on the 
mean energy loss by a factor of 2 . For more details see $[15,16]$ and references therein.

The system we study consists of a four-dipole chicane between rf cavities and quadrupoles; see Fig. 4. In this paper we limit our study to the chicane. The phase space density on entrance to the chicane is a smooth function $a_{0}\left(z, p_{z}, x, p_{x}\right)$ modulated by a factor $1+A \cos \left(2 \pi z / \lambda_{0}\right)$ where $A$ is a small amplitude and $\lambda_{0}$ is the perturbation wavelength. The function $a_{0}$ contains the energy chirp, the $z-p_{z}$ correlation that is necessary for bunch compression. The beam frame coordinates $\left(z, p_{z}, x, p_{x}\right)$ are standard and are defined in Sec. II B. Our initial phase space density for the MCP model is discussed in detail at the beginning of Sec. IV.

A standard approach to study the microbunching instability consists in calculating a gain factor for a given initial modulation wave number $k_{0}[12,13,17]$. This gain factor is defined as

$$
\text { (S) }\left(k_{0}\right)=\left|\tilde{\rho}\left[k_{0} C\left(s_{f}\right), s_{f}\right] / \tilde{\rho}\left(k_{0}, 0\right)\right|,
$$

where

$$
\tilde{\rho}(k, s)=\int_{\mathbb{R}} d z \exp (-i k z) \rho(z, s)
$$

for a given initial wavelength of $\lambda_{0}=2 \pi / k_{0}$. Here $\rho(z, s)$ is the longitudinal spatial density, $C(s)=1 /\left[1+h R_{56}(s)\right]$ is the compression factor of the chicane, $s$ is the arclength along the reference orbit, $s_{f}$ is the value of $s$ at the exit of the chicane, and $h$ is the linear chirp parameter. Apparently the motivation for this definition is the intuition that without self-fields an initial modulation at wave number $k_{0}$ will appear at wave number $k_{0} C\left(s_{f}\right)$ at the end of the chicane due to the bunch compression. We will show there are problems with this definition of the gain, by a detailed study of $\tilde{\rho}$ and its modulus $|\tilde{\rho}|$ in the full nonlinear calculation. We refer to $|\tilde{\rho}(\cdot, s)|$ as the Fourier spectrum of $\rho$ at $s$ or simply the spectrum at $s$.

The function $\tilde{\rho}(k, s)$ can be computed in the full nonlinear self-consistent scheme for the MCP model, but can also be approximated in some cases through a solution of a linearized Vlasov equation leading us to an analytical model. If the initial distribution is a coasting beam with linear energy chirp, and the collective force is given in terms of a 1D impedance or wake potential, this equation is equivalent to a $1 \mathrm{D}$ integral equation for $\tilde{\rho}\left[k_{0} C(s), s\right]$. This equation was derived by Heifets, Stupakov, and Krinsky [13] and also by Huang and Kim [12]. Determining an approximate solution of the equation by iteration, Huang and Kim found an explicit formula for the gain. We will refer to this as the analytical model whose initial phase space density is discussed at the beginning of Sec. IV.

Aiming to clarify the basis of the 1D integral equation, we rederive it starting in a general framework allowing a bunched beam and arbitrary chirp. The only restriction is the 1D Ansatz of the collective force. We linearize about an arbitrary solution of the nonlinear Vlasov equation, obtaining a $2 \mathrm{D}$ integral equation for $\tilde{\rho}(k, s)$, which reduces to the $1 \mathrm{D}$ equation for a coasting beam with linear chirp. A numerical treatment of the $2 \mathrm{D}$ equation seems interesting and feasible, but has not yet been done.

For the MCP model we compute the gain factor (1) with our MCP solver and compare it with the analytical gain factor as it was computed in [12]. Agreement is good at medium wavelengths, in spite of the fact that our collective force is computed in a more detailed way than that of [12], the latter being derived from the impedance for steady state CSR without account of finite magnet length. At long wavelengths we begin to see the breakdown of the coasting beam assumption. At short wavelengths, as little as $80 \mu \mathrm{m}$ in calculations to date, there are deviations from the analytical gain factor. The source of discrepancy has not yet been analyzed. It might be due to nonlinearity, or to the different models of the collective force, or both. As part of our comparison we discuss in detail the Fourier spectra $|\tilde{\rho}(k, s)|$ at $s=0$ and $s=s_{f}$ and their relation to the gain curve.

To define clearly our Vlasov-Maxwell starting point we begin with exact equations, but for practical work we later make approximations based on the following assumptions: (A) The maximum bunch size $\Delta$ is small compared to the minimum bending radius. (B) In beam frame coordinates the bunch form (and also the form of the phase space distribution) changes very little during a time $\Delta / c$. Correspondingly, the field of the bunch at a comoving point changes little on such a time interval. Here $\Delta$ is the biggest extent of the bunch in any direction. Under typical conditions (A) and (B) should be very well satisfied. We also assume that the beam is relativistic $(\gamma \gg 1)$, as is true in the example studied, but that assumption could be removed without great cost.

The paper is organized as follows. The introduction to Sec. II is devoted to a precise statement of our VlasovMaxwell system in laboratory coordinates. In Sec. II A, we derive our formula for the fields in terms of sources. We integrate the equations of motion in the beam frame and Sec. II B is devoted to the definition of the beam frame and beam coordinates, the beam frame equations of motion, and the transformation of densities from beam frame to laboratory frame which is needed to determine the laboratory frame sources. In Sec. II C, we give the details of our MCP algorithm. An important part of this section is the introduction of coordinates in which the charge density is essentially stationary without self-fields. Section III is devoted to the derivation of integral equations for determining $\tilde{\rho}$. It contains both our new 2D integral equation and the previously derived 1D integral equation. Section IV discusses the results of our numerical calculations. In addition to a detailed study of the Fourier spectra we also give examples of 2D charge densities and electromagnetic fields to show that we can calculate these con- 
fidently and that they have no visually discernible noise. A reader who is already familiar with the basic nomenclature and who is only interested in the results can go immediately to Sec. IV.

\section{VLASOV-MAXWELL SYSTEM}

Our basic starting point is the Vlasov-Maxwell system in $3 \mathrm{D}$, i.e., we assume collisions can be ignored and that the $N$-particle bunch can be approximated by a continuum. Our final scheme for computation is less ambitious, but we think that it might be a reasonable approximation to the full system. We reduce the problem from $3 \mathrm{D}$ to $2 \mathrm{D}$, since we expect that most of the acceleration by self-fields will be in the plane of the unperturbed orbit. We use a particle method that follows the charge density rather than the phase space density, but hope that with sufficient attention to smoothing the result approximates that defined by the Vlasov-Maxwell system.

Our coordinate system, $(Z, X, Y)$, is shown in Fig. 1. We assume an external force due to a magnetic field, $B_{\text {ext }}(Z) \mathbf{e}_{Y}$, in the $Y$ direction. We define a reference orbit, $\left[\mathbf{R}_{r}(s), 0\right]=\left[Z_{r}(s), X_{r}(s), 0\right]^{T}$, lying in the $Y=0$ plane, where $\left[\mathbf{R}_{r}\left(\beta_{r} u\right), 0\right]$, as a function of $u$, is a solution of the Lorentz equations for the magnetic field $B_{\text {ext }}(Z) \mathbf{e}_{Y}$, and $u=c t$. Here $s$ is arclength along the reference orbit. In Fig. 1 we sketch $\left[\mathbf{R}_{r}(s), 0\right]$ for a four-dipole magnetic chicane bunch compressor. Writing the self-fields as $\mathbf{E}=$ $\left(E_{Z}, E_{X}, E_{Y}\right)^{T}$ and $\mathbf{B}=\left(B_{Z}, B_{X}, B_{Y}\right)^{T}$, we focus on the evolution of $\mathcal{F}=\left(E_{Z}, E_{X}, B_{Y}\right)^{T}$ and take $\left(E_{Y}, B_{Z}, B_{X}\right)=$ 0 . The latter entails planar motion in the $Y=$ const planes. We model shielding by the vacuum chamber by taking $\mathcal{F}=0$ at $Y= \pm g$, where $h=2 g$ is the height of the vacuum chamber as shown in Fig. 1. Let $H(Y) \geq 0$ be the fixed vertical charge distribution, zero for $|Y|>g$ and with $\int_{-g}^{g} H(Y) d Y=1$. The coupled Vlasov-Maxwell

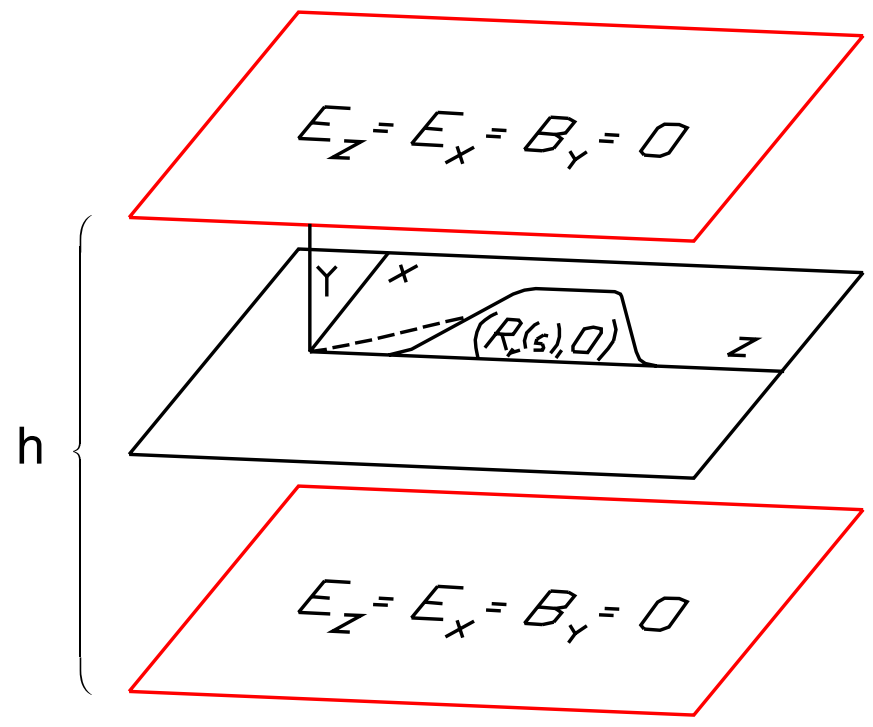

FIG. 1. (Color) Basic lab frame setup. system for the field vector $\mathcal{F}(\mathbf{R}, Y, u)$ and the phase space density $H(Y) \delta\left(P_{Y}\right) f_{L}(\mathbf{R}, \mathbf{P}, u)$, with the shielding boundary condition, takes the form

$$
\begin{gathered}
\square \mathcal{F}(\mathbf{R}, Y, u)=H(Y) \mathbf{S}(\mathbf{R}, u), \\
\partial_{u} f_{L}+\dot{\mathbf{R}} \cdot \nabla_{\mathbf{R}} f_{L}+\dot{\mathbf{P}} \cdot \nabla_{\mathbf{P}} f_{L}=0, \\
\mathcal{F}(\mathbf{R}, Y= \pm g, u)=0,
\end{gathered}
$$

where ${ }^{\cdot}=d / d u, \square=\partial_{Z}^{2}+\partial_{X}^{2}+\partial_{Y}^{2}-\partial_{u}^{2}$, and

$$
\begin{gathered}
\mathbf{S}(\mathbf{R}, u)=Z_{0} Q\left(\begin{array}{c}
c \partial_{Z} \rho_{L}+\partial_{u} J_{L, Z} \\
c \partial_{X} \rho_{L}+\partial_{u} J_{L, X} \\
\partial_{X} J_{L, Z}-\partial_{Z} J_{L, X}
\end{array}\right), \\
\dot{\mathbf{R}}=\frac{\mathbf{P}}{m \gamma(P) c}, \\
\dot{\mathbf{P}}=\frac{q}{c}\left\{\left[E_{Z}(\mathbf{R}, Y, u), E_{X}(\mathbf{R}, Y, u)\right]^{T}\right. \\
\left.+c\left[B_{\mathrm{ext}}(Z)+B_{Y}(\mathbf{R}, Y, u)\right](\dot{X},-\dot{Z})^{T}\right\} .
\end{gathered}
$$

Here $Z_{0}$ is the free space impedance, $Q$ is the total charge, $Q \rho_{L}(\mathbf{R}, u)$ is the $2 \mathrm{D}$ lab frame charge density (whence $\left.\int \rho_{L} d \mathbf{R}=1\right), Q H(Y)\left(J_{L, Z}, J_{L, X}, 0\right)^{T}(\mathbf{R}, u)$ is the $3 \mathrm{D}$ current density, $m$ is the electron rest mass, $q$ is the electron charge (so that $Q=N q$ where $N$ is the number of particles in the bunch), and $\gamma$ is the Lorentz factor. As will be explained in Sec. II A, instead of $\mathcal{F}$ we actually use the averaged field $\mathcal{F}_{L}=\left(\mathbf{E}_{L}, B_{L}\right)^{T}=\left(E_{L, Z}, E_{L, X}, B_{L}\right)^{T}$ so that instead of (8) we use

$$
\dot{\mathbf{P}}=\frac{q}{c}\left\{\mathbf{E}_{L}(\mathbf{R}, u)+c\left[B_{\mathrm{ext}}(Z)+B_{L}(\mathbf{R}, u)\right](\dot{X},-\dot{Z})^{T}\right\} .
$$

Equations (3) and (4) are completed by specifying $\mathbf{S}$ in terms of (6) and

$$
\begin{gathered}
\rho_{L}(\mathbf{R}, u)=\int_{\mathbb{R}^{2}} d \mathbf{P} f_{L}(\mathbf{R}, \mathbf{P}, u), \\
\mathbf{J}_{L}(\mathbf{R}, u)=\int_{\mathbb{R}^{2}} d \mathbf{P}[\mathbf{P} / m \gamma(P)] f_{L}(\mathbf{R}, \mathbf{P}, u),
\end{gathered}
$$

where $\mathbf{J}_{L}=\left(J_{L, Z}, J_{L, X}\right)^{T}$. Note that since $Q \rho_{L}$ is the charge density, $\rho_{L}$ is normalized whence

$$
1=\int_{\mathbb{R}^{2}} \rho_{L} d \mathbf{R}=\int_{\mathbb{R}^{4}} d \mathbf{R} d \mathbf{P} f_{L}(\mathbf{R}, \mathbf{P}, u) .
$$

We use $c, Z_{0}$ as our basic parameters instead of $\epsilon_{0}, \mu_{0}$, where $Z_{0}^{2}=\mu_{0} / \epsilon_{0}, \quad c^{2}=1 / \mu_{0} \epsilon_{0}$. We use SI units throughout.

\section{A. Field formula}

We calculate $\mathcal{F}$ produced by $\rho_{L}, \mathbf{J}_{L}$, but averaged over the $Y$ distribution: 


$$
\mathcal{F}_{L}(\mathbf{R}, u)=\langle\mathcal{F}(\mathbf{R}, \cdot, u)\rangle=\int_{-g}^{g} H(Y) \mathcal{F}(\mathbf{R}, Y, u) d Y .
$$

The averaging is appropriate, since we regard motion in the $Y$ direction as less important and do not allow it in computations. To evaluate (13) we begin with the general formula for $\mathcal{F}$, which follows from the retarded Green function for the 3D wave equation (3):

$$
\begin{aligned}
\mathcal{F}(\mathbf{R}, Y, u)= & -\frac{1}{4 \pi} \int_{\mathbb{R}^{2}} d \mathbf{R}^{\prime} \int_{\mathbb{R}} d Y^{\prime} \xi\left(Y^{\prime}\right) \\
& \times \frac{\mathbf{S}\left\{\mathbf{R}^{\prime}, u-\left[\left|\mathbf{R}^{\prime}-\mathbf{R}\right|^{2}+\left(Y^{\prime}-Y\right)^{2}\right]^{1 / 2}\right\}}{\left[\left|\mathbf{R}^{\prime}-\mathbf{R}\right|^{2}+\left(Y^{\prime}-Y\right)^{2}\right]^{1 / 2}} .
\end{aligned}
$$

Here $\xi(Y)$ is the effective vertical distribution needed to impose boundary conditions $[\mathcal{F}(Y= \pm g)=0]$ at the parallel plates by the method of images, namely $\xi(Y)=$ $\sum_{k=-\infty}^{\infty}(-1)^{k} H(Y-k h)$. We of course assume that the support of $H(Y)$ is well within the interval $(-g, g)$ and we also assumed in (14) that $H$ is even. The field for free space comes from the term with $k=0$.

In the calculations we take $u_{0}$ to be the time at which the front of the bunch enters the first magnet of the chicane. If $\mathbf{S}(\mathbf{R}, u)=0$ for $u \leq u_{0}$, then $\mathcal{F}(\mathbf{R}, Y, u)=0$ for $u \leq u_{0}$ and (14) becomes

$$
\begin{aligned}
\mathcal{F}(\mathbf{R}, Y, u)= & -\frac{1}{4 \pi} \int_{\mathcal{B}\left(\mathbf{R}, Y, u-u_{0}\right)} d \mathbf{R}^{\prime} d Y^{\prime} \xi\left(Y^{\prime}\right) \\
& \times \frac{\mathbf{S}\left\{\mathbf{R}^{\prime}, u-\left[\left|\mathbf{R}^{\prime}-\mathbf{R}\right|^{2}+\left(Y^{\prime}-Y\right)^{2}\right]^{1 / 2}\right\}}{\left[\left|\mathbf{R}^{\prime}-\mathbf{R}\right|^{2}+\left(Y^{\prime}-Y\right)^{2}\right]^{1 / 2}},
\end{aligned}
$$

where

$$
\begin{aligned}
\mathcal{B}\left(\mathbf{R}, Y, u-u_{0}\right) & :=\left\{\left(\mathbf{R}^{\prime}, Y^{\prime}\right):\left[\left|\mathbf{R}^{\prime}-\mathbf{R}\right|^{2}+\left(Y^{\prime}-Y\right)^{2}\right]^{1 / 2}\right. \\
& \left.\leq u-u_{0}\right\}
\end{aligned}
$$

for $u \geq u_{0}$. Furthermore $\mathcal{F}$ is the unique solution of the $3 \mathrm{D}$ wave equation (3) with the boundary condition (5) and initial condition $\mathcal{F}(\mathbf{R}, Y, u)=\partial_{u} \mathcal{F}(\mathbf{R}, Y, u)=0$ at $u=$ $u_{0}$ (see [18], p. 82). Of course, the source $\mathbf{S}(\mathbf{R}, u)$ is not zero for $u<u_{0}$, but the longitudinal field it produces is very small, $O\left(1 / \gamma^{2}\right)$, for a relativistic bunch on a straight path (pancake effect). Thus the self-fields in the chicane are essentially the same as if we merely put the source equal to zero for $u<u_{0}$. For nonrelativistic particles, one should start the integral at a much earlier time, allowing the bunch to feel its own space charge field prior to entrance of the first magnet. In the following $\mathcal{F}$ is given by (14) with the source zero for $u \leq u_{0}$. The specific value of $u_{0}$ is discussed in Sec. II C.

To average the field as in (13) we put $\eta=Y^{\prime}-Y$ and find

$$
\begin{aligned}
\mathcal{F}_{L}(\mathbf{R}, u)= & -\frac{1}{4 \pi} \int_{\mathbb{R}^{2}} d \mathbf{R}^{\prime} \int_{\mathbb{R}} d \eta \Phi(\eta) \\
& \times \frac{\mathbf{S}\left\{\mathbf{R}^{\prime}, u-\left[\left|\mathbf{R}^{\prime}-\mathbf{R}\right|^{2}+\eta^{2}\right]^{1 / 2}\right\}}{\left[\left|\mathbf{R}^{\prime}-\mathbf{R}\right|^{2}+\eta^{2}\right]^{1 / 2}},
\end{aligned}
$$

where $\Phi(\eta)=\int_{-g}^{g} H(Y) \xi(Y+\eta) d Y$. For a Gaussian $H(Y)$ with rms width $\sigma_{Y}$, we suppose that $\sigma_{Y} \ll g$ and obtain

$$
\begin{aligned}
\Phi(\eta) & =\sum_{k=-\infty}^{\infty} \frac{(-1)^{k}}{\sqrt{2 \pi} \sigma} \exp \left[-\frac{1}{2}\left(\frac{\eta-k h}{\sigma}\right)^{2}\right], \\
\sigma & =\sqrt{2} \sigma_{Y} .
\end{aligned}
$$

We assume that $\sigma$ is sufficiently small to justify replacing the Gaussians in (18) by $\delta(\eta-k h)$. We then have just a 2D integral, which will be the basis for our numerical work:

$$
\begin{aligned}
\mathcal{F}_{L}(\mathbf{R}, u)= & -\frac{1}{4 \pi} \sum_{k=-\infty}^{\infty}(-1)^{k} \int_{\mathbb{R}^{2}} d \mathbf{R}^{\prime} \\
& \times \frac{\mathbf{S}\left\{\mathbf{R}^{\prime}, u-\left[\left|\mathbf{R}^{\prime}-\mathbf{R}\right|^{2}+(k h)^{2}\right]^{1 / 2}\right\}}{\left[\left|\mathbf{R}^{\prime}-\mathbf{R}\right|^{2}+(k h)^{2}\right]^{1 / 2}} .
\end{aligned}
$$

Note that if $H(Y)=\delta(Y)$ then, for $Y=0$, (14) becomes (19), i.e., in this case $\mathcal{F}_{L}(\mathbf{R}, u)=\mathcal{F}(\mathbf{R}, 0, u)$. In fact, if $H(Y)=\delta(Y)$, then the averaging procedure not only gives the exact field (19) at $Y=0$ but also the exact $\left(E_{Y}, B_{Z}, B_{X}\right)$ vanishes at $Y=0$. With certain reasonable approximations it seems possible to retain a nonzero vertical spread while maintaining a reduction to a $2 \mathrm{D}$ integration. The accuracy of such a reduction is still under investigation.

The integration in (19) is restricted to a very small part of $\mathbb{R}^{2}$, because of the small size of the bunch, but it is awkward to locate this region owing to the fact that spatial and temporal arguments of the source both depend on $\mathbf{R}^{\prime}$. The task of integration is greatly simplified if we take the temporal argument to be a new variable of integration. We first transform to polar coordinates $(\chi, \theta)$, then take the temporal argument $v$ in place of the radial coordinate $\chi$. That is,

$$
\begin{gathered}
\mathbf{R}^{\prime}-\mathbf{R}=\chi \mathbf{e}(\theta), \quad \mathbf{e}(\theta)=(\cos \theta, \sin \theta)^{T}, \\
v=u-\left[\chi^{2}+(k h)^{2}\right]^{1 / 2} .
\end{gathered}
$$

This conveniently gets rid of the potentially small divisor in (19), giving the field simply as an integral over the source:

$$
\begin{aligned}
\mathcal{F}_{L}(\mathbf{R}, u)= & -\frac{1}{2 \pi} \sum_{k=0}^{\infty}(-1)^{k}\left(1-\delta_{k 0} / 2\right) \\
& \times \int_{u_{0}}^{u-k h} d v \int_{-\pi}^{\pi} d \theta \mathbf{S}[\tilde{\mathbf{R}}(\theta, v ; u), v],
\end{aligned}
$$

where $\tilde{\mathbf{R}}(\theta, v ; u)=\mathbf{R}+\left[(u-v)^{2}-(k h)^{2}\right]^{1 / 2} \mathbf{e}(\theta)$.

To estimate the effective region of the $\theta$ integration in (21), note that the source in (21) has significant values only 
for $\tilde{\mathbf{R}}(\theta, v ; u)$ restricted to a bunch-sized neighborhood of $\mathbf{R}_{r}\left(\beta_{r} v\right)$; i.e., the bunch is close to the reference particle. For the field $\mathcal{F}_{L}$ at time $u$ we are interested only in $\mathbf{R}$ in a bunch-sized neighborhood of $\mathbf{R}_{r}\left(\beta_{r} u\right)$. Thus for $\mathbf{R}$ in a small neighborhood of $\mathbf{R}_{r}\left(\beta_{r} u\right)$ the integrand is appreciable only when

$$
\begin{aligned}
\left|\tilde{\mathbf{R}}(\theta, v ; u)-\mathbf{R}_{r}\left(\beta_{r} v\right)\right| \approx & \mid \mathbf{R}_{r}\left(\beta_{r} u\right)-\mathbf{R}_{r}\left(\beta_{r} v\right) \\
& +\left[(u-v)^{2}-(k h)^{2}\right]^{1 / 2} \\
& \times \mathbf{e}(\theta) \mid=O(\Delta),
\end{aligned}
$$

where $\Delta$ was introduced in Sec. I. For $k=0$ and $u-v$ large compared to $\Delta$, this cannot be satisfied unless $\mathbf{e}(\theta)$ has nearly the same direction as $\mathbf{R}_{r}\left(\beta_{r} u\right)-\mathbf{R}_{r}\left(\beta_{r} v\right)$, which is to say that the domain of $\theta$ integration is tiny (and close to $\theta=0$ for a chicane with small bending angle). When $u-v$ gets close to $\Delta$ the domain expands precipitously to the full $[-\pi, \pi]$. For $k \neq 0$ the condition (22) cannot be met unless $u-v \gg k h$, so for image charges there are no contributions to the $v$ integral close to its upper limit.

The $\theta$ integration is over an arc centered at the observation point $\mathbf{R}$ at time $u$ with radius $\sqrt{(u-v)^{2}-(k h)^{2}}$, its extent being its intersection with the bunch at time $v$. This is illustrated in the Fig. 2 for $k=0$. When $v$ is close to $u$ the source bunch and the observation region (the region of the bunch at time $u$ ) overlap and the $\theta$ support of the source is large. However, for most $v$ the $\theta$ support is small and it is important to determine the approximate support as shown in the figure. Currently, the $\theta$ integration is done with the trapezoidal rule, which is superconvergent. The remaining $v$ integrand varies with $v, \mathbf{R}$, and $u$ in ways we have not yet quantified and so we use an adaptive integrator.

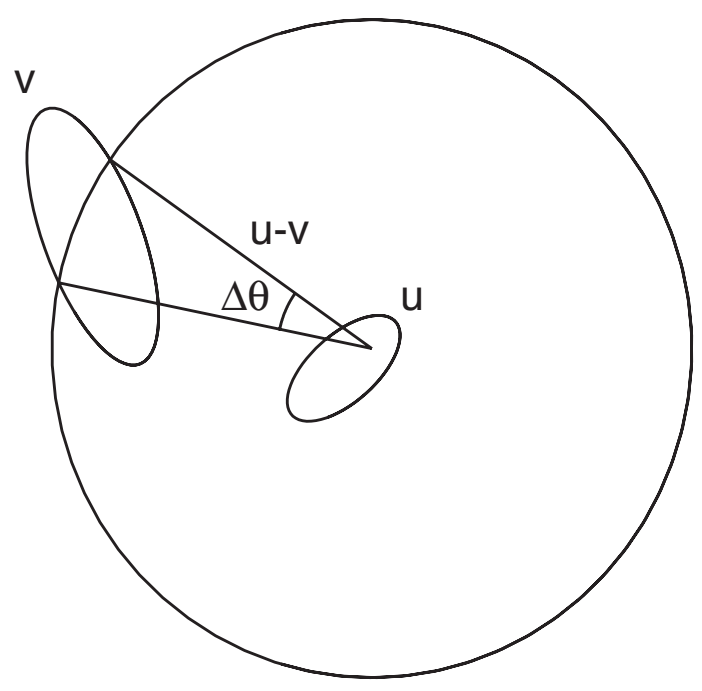

FIG. 2. Plan for $\theta$ integration.

\section{B. Beam frame}

In our approach the Maxwell equations are solved in the lab frame but the equations of motion are integrated in the beam frame. Here we discuss the beam frame coordinates and the transformation of the densities between the two frames.

The beam frame is defined in terms of the reference orbit $\mathbf{R}_{r}(s)=\left[Z_{r}(s), X_{r}(s)\right]^{T}$ which in turn is defined by the Lorentz equations without self-fields. We take $s=0$ at the entrance of the chicane, i.e., $\mathbf{R}_{r}(0)$ is the entry point of the reference orbit into the chicane. The unit tangent vector, $\mathbf{t}$, to the reference orbit is just $\mathbf{t}(s)=\mathbf{R}_{r}^{\prime}(s)$ and we define the unit normal vector, $\mathbf{n}$, by $\mathbf{n}(s)=$ $\left[-X_{r}^{\prime}(s), Z_{r}^{\prime}(s)\right]^{T}$ so that $\mathbf{n}$ is a $\pi / 2$ counterclockwise rotation from $\mathbf{t}$ as shown in Fig. 3. It follows from the Lorentz equations that $\mathbf{t}^{\prime}(s)=-q B_{\text {ext }}\left[Z_{r}(s)\right] \mathbf{n}(s) / P_{r}$, where $P_{r}=$ $m \gamma_{r} \beta_{r} c$ is the momentum of the reference particle. This determines the curvature $\kappa$ up to a sign and we choose $\kappa(s)=q B_{\text {ext }}\left[Z_{r}(s)\right] / P_{r}$. Thus $\mathbf{t}^{\prime}(s)=-\kappa(s) \mathbf{n}(s)$ and $\mathbf{n}^{\prime}(s)=\kappa(s) \mathbf{t}(s)$. In terms of Fig. 1 this makes $\kappa$ negative in the first magnet, positive in the second magnet and so on.

The beam frame Frenet-Serret coordinates are $s, x$, where $s$ is the arclength along the reference orbit and $x$ is the perpendicular distance along $\mathbf{n}$. Thus the transformation from $(Z, X)$ to $(s, x)$ is

$$
\mathbf{R}=\mathbf{R}_{r}(s)+x \mathbf{n}(s) .
$$

In addition, we define $p_{s}$ and $p_{x}$ by $\mathbf{P}=P_{r}\left[p_{s} \mathbf{t}(s)+\right.$ $\left.p_{x} \mathbf{n}(s)\right]$.

Our lab to beam transformation has three steps:

$$
\begin{aligned}
\left(Z, P_{Z}, X, P_{X} ; u\right) & \rightarrow\left(s, p_{s}, x, p_{x} ; u\right) \rightarrow\left(u, p_{s}, x, p_{x} ; s\right) \\
& \rightarrow\left(z, p_{z}, x, p_{x} ; s\right) .
\end{aligned}
$$

The first step is the transformation just discussed. In the second step the variables $s$ and $u$ are interchanged making

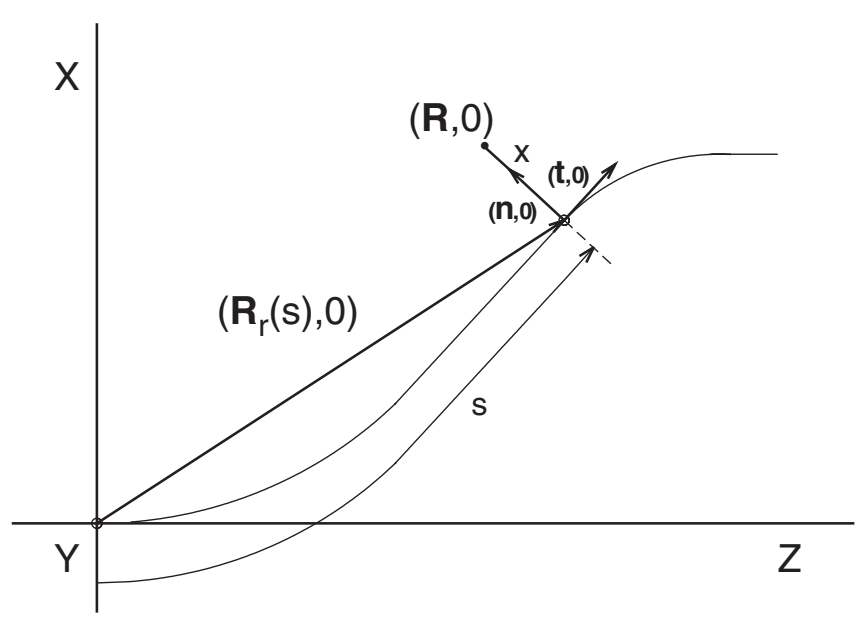

FIG. 3. Beam frame coordinates. 
$s$ the new independent variable. In the final step $z=s-$ $\beta_{r} u$ replaces $u$ as a dependent variable and $p_{z}=(\gamma-$ $\left.\gamma_{r}\right) / \gamma_{r}$ replaces $p_{s}$. Thus the variables $z, p_{z}, x, p_{x}$ are small near the reference orbit which corresponds to $z=x=0$. Equation (23) defines $s=s(\mathbf{R})$ and $x=x(\mathbf{R})$ in a neighborhood of the reference orbit so that $z=z(\mathbf{R}, u)=$ $s(\mathbf{R})-\beta_{r} u$ and we have the identity $\mathbf{R} \equiv \mathbf{R}_{r}[z(\mathbf{R}, u)+$ $\left.\beta_{r} u\right]+x(\mathbf{R}) \mathbf{n}\left[z(\mathbf{R}, u)+\beta_{r} u\right]$. Since $z$ is small for $\mathbf{R}$ in the bunch, expanding for small $z$ gives $\mathbf{R}=\mathbf{R}_{r}\left(\beta_{r} u\right)+$ $M\left(\beta_{r} u\right) \mathbf{r}+O\left(\kappa z^{2}, \kappa x z\right)$ and we obtain the approximate inverse

$$
\begin{gathered}
\mathbf{r}=M^{T}\left(\beta_{r} u\right)\left[\mathbf{R}-\mathbf{R}_{r}\left(\beta_{r} u\right)\right], \quad M(s)=[\mathbf{t}(s), \mathbf{n}(s)], \\
\mathbf{r}=(z, x)^{T} .
\end{gathered}
$$

We make extensive use of formula (25) within its domain of validity, namely, when $\mathbf{R}$ is in a neighborhood of $\mathbf{R}_{r}\left(\beta_{r} u\right)$ comparable in extent to the bunch size, and the bunch size is small compared to the bending radius [see Assumption A of Sec. I whereby $\kappa \Delta \ll 1$ whence $\kappa z \ll 1$, $\kappa x \ll 1$ so that the $O$ term above (25) can be neglected].

The equations of motion in $\left(z, p_{z}, x, p_{x} ; s\right)$ have the fields $\mathcal{F}_{L}(\mathbf{R}, u)$ evaluated at $\mathbf{R}=\mathbf{R}_{r}(s)+x \mathbf{n}(s)$ and $u=(s-$ $z) / \beta_{r}$. We have the following approximations:

$$
\begin{aligned}
& \mathcal{F}_{L}\left[\mathbf{R}_{r}(s)+x \mathbf{n}(s),(s-z) / \beta_{r}\right] \\
& \quad \approx \mathcal{F}_{L}\left[\mathbf{R}_{r}(s+z)+x \mathbf{n}(s+z), s\right] \\
& \quad \approx \mathcal{F}_{L}\left[\mathbf{R}_{r}(s)+M(s) \mathbf{r}, s\right] .
\end{aligned}
$$

At the first approximation we use the fact that the fields are slowly varying in $s$ for fixed $\mathbf{r}$ (see Assumption B of Sec. I) and that $\beta_{r} \approx 1$. The second approximation uses the fact that we are only interested in the fields in the bunch for $\mathbf{r}$ small [this again uses assumption A of Sec. I and drops the $O$ term above (25)]. From (7) and (9) it is possible to deduce

$$
\begin{array}{cc}
z^{\prime}=-\kappa(s) x & p_{z}^{\prime}=F_{z 1}(\hat{\mathbf{R}}, s)+p_{x} F_{z 2}(\hat{\mathbf{R}}, s) \\
x^{\prime}=p_{x} & p_{x}^{\prime}=\kappa(s) p_{z}+F_{x}(\hat{\mathbf{R}}, s),
\end{array}
$$

where $\hat{\mathbf{R}}=(\hat{Z}, \hat{X})^{T}=\hat{\mathbf{R}}(\mathbf{r}, s)=\mathbf{R}_{r}(s)+M(s) \mathbf{r}$ and ${ }^{\prime}=$ $d / d s$. The collective forces are given approximately by

$$
\begin{aligned}
F_{z 1}(\hat{\mathbf{R}}, s)= & \frac{q}{P_{r} c} \mathbf{E}_{L}(\hat{\mathbf{R}}, s) \cdot \mathbf{t}(s), \\
F_{z 2}(\hat{\mathbf{R}}, s)= & \frac{q}{P_{r} c} \mathbf{E}_{L}(\hat{\mathbf{R}}, s) \cdot \mathbf{n}(s) \\
F_{x}(\hat{\mathbf{R}}, s)= & \frac{q}{P_{r} c}\left\{-E_{L, Z}(\hat{\mathbf{R}}, s) X_{r}^{\prime}(s)+E_{L, X}(\hat{\mathbf{R}}, s) Z_{r}^{\prime}(s)\right. \\
& \left.-c B_{L}(\hat{\mathbf{R}}, s)\right\} .
\end{aligned}
$$

We have expanded $F_{x}$ in order to point out that each of the last two terms are large whereas their difference is small. Details will be presented in [19].
The equations of motion (27), without the self-fields, represent the Lorentz equations in linearized form for the relativistic case $\gamma_{r} \gg 1$. Including the self-fields we write the initial value problem for (27) as

$$
\zeta^{\prime}=A(s) \zeta+G\left(\zeta, s ; \mathcal{F}_{L}\right), \quad \zeta(0)=\zeta_{0},
$$

where $\zeta=\left(z, p_{z}, x, p_{x}\right)^{T}$. The linear part $\zeta^{\prime}=A(s) \zeta$ can be solved and the solution written $\zeta=\Phi(s, 0) \zeta_{0}$, $\Phi(0,0)=I_{4 \times 4}$. Here $\Phi(s, \tau)$ is the transfer map (principal solution matrix) which is defined in terms of the dispersion function, $D(s, \tau)$, and momentum compaction function, $R_{56}(s, \tau)$, in Sec. III B. The equations of motion in the interaction picture become

$$
\zeta_{0}^{\prime}=\Phi(0, s) \mathcal{G}\left[\Phi(s, 0) \zeta_{0}, s ; \mathcal{F}_{L}\right]
$$

We have found that it is numerically more efficient to integrate (30) than to integrate (27).

Our field formula is in the lab frame so the lab charge and current densities must be determined from the beam frame phase space density. The relation between the lab frame phase space density, $f_{L}$, and the beam frame phase space density, $f$, is

$$
f_{L}\left(Z, P_{Z}, X, P_{X}, u\right)=\frac{\beta_{r}^{2}}{P_{r}^{2}} f\left(z, p_{z}, x, p_{x}, s\right) .
$$

Here $f$ is normalized, i.e.,

$$
1=\int_{\mathbb{R}^{4}} d z d p_{z} d x d p_{x} f\left(z, p_{z}, x, p_{x}, s\right),
$$

as is $f_{L}$ in (12). Equation (31) leads to

$$
\begin{gathered}
\rho_{L}(\mathbf{R}, u) \approx \int_{\mathbb{R}^{2}} d p_{z} d p_{x} f=\rho_{B}(\mathbf{r}, s), \\
\mathbf{J}_{L}(\mathbf{R}, u) \approx \beta_{r} c\left[\rho_{B}(\mathbf{r}, s) \mathbf{t}(s)+\tau_{B}(\mathbf{r}, s) \mathbf{n}(s)\right],
\end{gathered}
$$

where $\tau_{B}(\mathbf{r}, s)=\int_{\mathbb{R}^{2}} p_{x} f\left(z, p_{z}, x, p_{x}, s\right) d p_{z} d p_{z}$. Note that since $\int_{\mathbb{R}^{2}} \rho_{B}(z, x, s) d z d x=1, \rho_{B}$ is the beam frame spatial density and $Q \rho_{B}$ is the beam frame charge density. The main function in the definition (1) of the gain factor is $\tilde{\rho}$ in (2) where

$$
\rho(z, s)=\int_{\mathbb{R}} d x \rho_{B}(z, x, s)=\int_{\mathbb{R}^{3}} d p_{z} d x d p_{x} f(\zeta, s) .
$$

Using the fact that $f\left(z, p_{z}, x, p_{x}, \cdot\right)$ is slowly varying and $\rho_{B}(\mathbf{r}, s)$ has its support for $\mathbf{r}$ small, we have $\rho_{B}\left[z(\mathbf{R}, u), x(\mathbf{R}, u), z(\mathbf{R}, u)+\beta_{r} u\right] \approx \rho_{B}\left(\hat{\mathbf{r}}, \beta_{r} u\right)$, where $\hat{\mathbf{r}}=M^{T}\left(\beta_{r} u\right)\left[\mathbf{R}-\mathbf{R}_{r}\left(\beta_{r} u\right)\right]$. Thus

$$
\begin{gathered}
\rho_{L}(\mathbf{R}, u) \approx \rho_{B}\left(\hat{\mathbf{r}}, \beta_{r} u\right) \\
\mathbf{J}_{L}(\mathbf{R}, u) \approx \beta_{r} c\left[\rho_{B}\left(\hat{\mathbf{r}}, \beta_{r} u\right) \mathbf{t}\left(z+\beta_{r} u\right)\right. \\
\left.+\tau_{B}\left(\hat{\mathbf{r}}, \beta_{r} u\right) \mathbf{n}\left(z+\beta_{r} u\right)\right],
\end{gathered}
$$

where the $\mathbf{J}_{L}$ approximation is derived similarly to that for $\rho_{L}$. 
There are subtleties in the second transformation of (24) caused by interchanging the roles of $u$ and $s$ as independent and dependent variables. The phase space density transformation (31), the normalization (32), and the approximations will be discussed in detail in [19].

\section{A method of solution: Monte Carlo particle method}

We have discussed our method for calculating the fields in the lab frame and the determination of the lab frame charge and current densities from the beam frame phase space density. Here we discuss a method of solution of the coupled Vlasov-Maxwell system similar to traditional particle methods, variously called particle-in-cell (PIC) or macroparticle methods. We call it the Monte Carlo particle (MCP) method, because it uses a Monte Carlo method to determine a smooth charge distribution from an ensemble of particles.

Before we developed the MCP method we considered solving the Vlasov equation using the method of local characteristics (or "semi-Lagrangian method"), which has been extremely effective in problems with a 2D phase space. This deals with the Vlasov equation in a very direct way, defining the phase space density by its values on a grid with interpolation to off-grid points. The density is updated by integrating backward from grid points, with the collective force regarded as constant during a time step. Since the backward orbits land at off-grid points, this update requires interpolation. In comparison with usual particle methods, this method offers much lower noise and the possibility of a relatively direct control of accuracy by monitoring interpolation error. On the other hand, it is relatively expensive in computation time and memory, and in the case of bunch compressors it is technically complicated because the density is concentrated in a narrow region of phase space that evolves in time in a manner that is not known a priori [20]. We are studying ways to deal with this evolving support, since it would be inefficient to use many grid points where the density is negligibly small. Possible techniques include changes of variable [2], an evolving selection of fiducial grid points, and the use of forward characteristics rather than backward [21]. Although we have high hopes for success in this direction, the present report has the more modest goal of improving the particle method, in which it is much easier to deal with the support question since one has to work only with the charge density in $2 \mathrm{D}$ rather than the phase space density in $4 \mathrm{D}$. In particle methods the connection to the Vlasov equation is unfortunately indirect, and the control of accuracy relies entirely on the experiment of increasing the number of particles. Even if one believes that a solution of the Vlasov equation is obtained in the limit, it is usually too expensive to make a convincing empirical demonstration of convergence.

In the Monte Carlo particle method we represent the charge/current density in the beam frame as a truncated
Fourier series, thus giving ourselves a density that is smooth, of class $C^{\infty}$. The integrals representing the Fourier coefficients are evaluated by Monte Carlo sampling of the integrand, over the ensemble of particles. Ideally, one would use the resulting Fourier series and its gradient to evaluate the source in the field formula. That is too expensive, however, since it involves multiple summations of the Fourier series, at points not amenable to the fast Fourier transform. Instead, we use the Fourier series to put the density and its gradient on a grid, and then use low order polynomial interpolation for evaluations at off-grid points. Thus we accomplish something similar to charge deposition in particle-in-cell codes, but by a different route, and get the gradient as well as the density itself at grid points. Our method gives low noise, but is costly at high levels of resolution. We have not yet carried out a careful comparison with more usual methods at similar levels of cost and resolution.

It is cost effective to make an $s$-dependent coordinate transformation so that the 2D spatial density can be accurately represented in a grid which does not depend on $s$. Since in our studies the uncorrelated energy spread $\sigma_{u}$ and the spread in the transverse momentum $\sigma_{p x_{0}}$ at entrance of the chicane are small, we found that the coordinate transformation $(z, x) \leftrightarrow(\tilde{z}, \tilde{x})$ via

$$
z=\left[1+h R_{56}(s)\right] \tilde{z}-D^{\prime}(s) \tilde{x}, \quad x=h D(s) \tilde{z}+\tilde{x}
$$

gives an almost stationary situation, where particles are at rest in the limit of no self-fields, and $\sigma_{u}=0$ and $\sigma_{p x_{0}}=0$. Here $h$ is the chirp parameter and $D(s)=D(s, 0)$ and $R_{56}(s)=R_{56}(s, 0)$ where $D(s, \tau)$ and $R_{56}(s, \tau)$ are defined in (84) and (85). The transformation (38) is obtained solving (29) without self-fields and with initial conditions $z=$ $\tilde{z}, p_{z}=h \tilde{z}, x=\tilde{x}$, and $p_{x}=0$. Since we estimate the charge density with a Fourier expansion in the unit square $[0,1] \times[0,1]$, our final grid transformation $(\tilde{z}, \tilde{x}) \leftrightarrow$ $\left(x_{1}, x_{2}\right)$ is obtained by a simple scaling and translation. For the MCP model in Sec. IV, we show the evolution of the spatial density both with and without self-fields in the coordinates $\left(x_{1}, x_{2}\right)$ with the spatial density, $\rho_{g}$, defined on our $s$-independent grid which is explained in that section.

We now describe our algorithm more concretely. To generate the initial positions of $\mathcal{N}$ particles at $s=0$ we use the acceptance-rejection method [22], assuming particles are independent identically distributed (IID) according to the initial phase space density. Since the reference particle corresponds to $z=0$ and since $z=s-\beta_{r} u$, the reference particle arrives at the chicane entrance at $u=0$. At $s=0$ our bunch effectively has $z$ supported in $(-a, a)$ where the longitudinal size parameter $a$ of the MCP model is explained in Sec. IV. Thus the particle at the head of the bunch arrives at $s=0$ at the time $-a / \beta_{r}$ and we take the latter to be $u_{0}$ whence at $u=u_{0}$ the particles have $z$ coordinates in the interval $(-2 a, 0)$. The field formula (21) can now be applied. 
For a small step $s \rightarrow s+\Delta s$ we proceed as follows.

1. Denoting $\rho_{B}, \tau_{B}$ in the grid coordinates $\left(x_{1}, x_{2}\right)$ by $\rho_{g}$, $\tau_{g}$, respectively, we expand $\rho_{g}\left(x_{1}, x_{2}, s\right)$ and $\tau_{g}\left(x_{1}, x_{2}, s\right)$ in a finite Fourier series:

$$
\begin{gathered}
\rho_{g}\left(x_{1}, x_{2}, s\right)=\sum_{i=0}^{I} \sum_{j=0}^{J} \theta_{i j}(s) \phi_{i}\left(x_{1}\right) \phi_{j}\left(x_{2}\right), \\
\tau_{g}\left(x_{1}, x_{2}, s\right)=\sum_{i=0}^{I} \sum_{j=0}^{J} \Theta_{i j}(s) \phi_{i}\left(x_{1}\right) \phi_{j}\left(x_{2}\right),
\end{gathered}
$$

where

$$
\begin{aligned}
& \theta_{i j}(s)=\int_{A} d x_{1} d x_{2} \phi_{i}\left(x_{1}\right) \phi_{j}\left(x_{2}\right) \rho_{g}\left(x_{1}, x_{2}, s\right), \\
& \Theta_{i j}(s)=\int_{A} d x_{1} d x_{2} \phi_{i}\left(x_{1}\right) \phi_{j}\left(x_{2}\right) \tau_{g}\left(x_{1}, x_{2}, s\right) .
\end{aligned}
$$

Here $\left\{\phi_{i}\right\}$ is the orthonormal basis $\phi_{0}(x)=1$ and $\phi_{i}(x)=$ $\sqrt{2} \cos (i \pi x)$ for $i \geq 1, x \in[0,1]$. Note that $\rho_{g}$ is now the actual spatial density, in the coordinates $x_{1}, x_{2}$, with nonzero $\sigma_{u}$ and $\sigma_{p_{x 0}}$ and with self-fields.

Since $\rho_{g}$ is a probability density the Fourier coefficients $\theta_{i j}$ may be written as the expected value $E$ of $\phi_{i}\left(X_{1}\right) \phi_{j}\left(X_{2}\right)$ with respect to $\rho_{g}(\cdot, s)$

$$
\begin{aligned}
\theta_{i j}(s) & =E\left\{\phi_{i}\left(X_{1}\right) \phi_{j}\left(X_{2}\right)\right\} \\
& =\int_{A} d x_{1} d x_{2} \phi_{i}\left(x_{1}\right) \phi_{j}\left(x_{2}\right) \rho_{g}\left(x_{1}, x_{2}, s\right),
\end{aligned}
$$

where $X=\left(X_{1}, X_{2}\right)$ is the random variable with probability density $\rho_{g}$. To estimate $\tau_{g}$, which is not a probability density, we notice that the Fourier coefficients $\Theta_{i j}$ may be written as the expected value $E$ of $\phi_{i}\left(X_{1}\right) \phi_{j}\left(X_{2}\right) P_{X}$ with respect to $f_{g}(\cdot, s)$

$$
\begin{aligned}
\Theta_{i j}(s)= & E\left\{\phi_{i}\left(X_{1}\right) \phi_{j}\left(X_{2}\right) P_{X}\right\} \\
= & \int_{A} d x_{1} d x_{2} \int_{\mathbb{R}^{2}} d p_{z} d p_{x} \phi_{i}\left(x_{1}\right) \phi_{j}\left(x_{2}\right) \\
& \times p_{x} f_{g}\left(x_{1}, p_{z}, x_{2}, p_{x}, s\right),
\end{aligned}
$$

where $X=\left(X_{1}, P_{Z}, X_{2}, P_{X}\right)$ is the random variable with probability density $f_{g}(\cdot, s)$. It follows that the natural estimate of $E$ is the sample mean, i.e., we have the following two Monte Carlo formulas:

$$
\begin{gathered}
\theta_{i j}(s) \approx \frac{1}{\mathcal{N}} \sum_{n=1}^{\mathcal{N}} \phi_{i}\left(X_{1 n}\right) \phi_{j}\left(X_{2 n}\right), \\
\Theta_{i j}(s) \approx \frac{1}{\mathcal{N}} \sum_{n=1}^{\mathcal{N}} \phi_{i}\left(X_{1 n}\right) \phi_{j}\left(X_{2 n}\right) P_{X n},
\end{gathered}
$$

where a realization of the random variable $X=$ $\left(X_{1}, P_{Z}, X_{2}, P_{X}\right)$ is obtained from beam frame scattered phase space points $z_{i}, p_{z i}, x_{i}, p_{x i}$ at $s, i=1, \ldots, \mathcal{N}$ [via the transformation: $\left.\left(z_{i}, p_{z_{i}}, x_{i}, p_{x_{i}}\right) \rightarrow\left(x_{1 i}, p_{z_{i}}, x_{2 i}, p_{x_{i}}\right)\right]$. This is a density estimation used in statistical estimation, see, e.g., [23]. The Monte Carlo computation is done in parallel, i.e., the sums in (45) and (46) are each split into $N_{p}$ pieces where $N_{p}$ is the number of processors. In other words, each processor only computes the sum over $\mathcal{N} / N_{p}$ terms in (45) and (46). In Sec. IV we discuss how we determine $\mathcal{N}$ and $(I, J)$ for a particular simulation.

2. The force fields $\mathbf{E}_{L}(\mathbf{R}, s) \cdot \mathbf{t}(s), \mathbf{E}_{L}(\mathbf{R}, s) \cdot \mathbf{n}(s)$, $F_{x}(\mathbf{R}, s)$, which are needed in (28), are computed on the $s$-independent grid defined above. That is, given a grid point $\left(x_{1}, x_{2}\right)$, we compute the associated beam frame value $x$, then compute $\mathbf{R}_{r}(s)+x \mathbf{n}(s)$. The force fields can then be determined at these $\mathbf{R}$ values from $\mathcal{F}_{L}(\mathbf{R}, s)$. Using (21), with $k=0$, we have

$$
\mathcal{F}_{L}(\mathbf{R}, s)=-\frac{1}{4 \pi} \int_{u_{0}}^{s} d v \int_{-\pi}^{\pi} d \theta \mathbf{S}[\tilde{\mathbf{R}}(\theta, v ; s), v],
$$

where

$$
\tilde{\mathbf{R}}(\theta, v ; s)=\mathbf{R}+(s-v) \mathbf{e}(\theta) .
$$

Here we have taken $k=0$, since for the parameters used for the MCP model in Sec. IV it is found that shielding is not important. For some designs shielding could well play a role, so our code allows it to be included.

To do the double integral in (47) we apply a Gauss-Kronrad adaptive algorithm to the outer integral. Gauss-Kronrod picks a $v$ and then we determine the $\theta$ support, $\left(\theta_{\min }, \theta_{\max }\right)$. The inner $\theta$ integral is then done with the trapezoidal rule on a uniform mesh. For each point $[\tilde{\mathbf{R}}(\theta, v ; s), v]$ of demand the source value $\mathbf{S}[\tilde{\mathbf{R}}(\theta, v ; s), v]$ is determined by a triquadratic interpolation of $\mathbf{S}$ values. We notice that the Fourier method of item 1 not only gives an analytical representation at $s$ of $\rho_{g}$ and $\tau_{g}$ but of $\nabla \rho_{g}$ and $\nabla \tau_{g}$ as well. A representation of $\partial \rho_{g} / \partial s$ and $\partial \tau_{g} / \partial s$ is obtained by differentiating the Fourier coefficients with a finite difference scheme. Even though it is possible to construct the source term $\mathbf{S}$ by storing the "history" of the Fourier coefficients, i.e. $\theta_{i j}$ and $\Theta_{i j}, d \theta_{i j} / d s$ and $d \Theta_{i j} / d s$ on a grid in $s$, we found it is more efficient to store $\rho_{g}, \nabla \rho_{g}$, and $\partial \rho_{g} / \partial s$ (the same for $\tau_{g}$ ) on a 3D grid in $\left(x_{1}, x_{2}, s\right)$. We use a uniform grid in $\left(x_{1}, x_{2}, s\right)$. Note that the field computation is done in parallel by letting each processor compute $\mathbf{E}_{L} \cdot \mathbf{t}, \mathbf{E}_{L} \cdot \mathbf{n}, F_{x}$ on only $N_{g} / N_{p}$ grid points $\left(x_{1}, x_{2}\right)$ where $N_{p}$ is the number of processors and $N_{g}$ is the number of grid points.

3 . We use item 2 to push the particles in the interaction picture of (30). This allows us to use an Euler scheme where the integration step $\Delta s$ is determined by the strength and smoothness of the self-fields. The force fields have been calculated on a grid in $\left(x_{1}, x_{2}\right)$ as outlined in item 2 above. To calculate the fields at particle positions needed in (28) we use a biquadratic interpolation. The particle push- 
ing is done in parallel, i.e., each processor only pushes $\mathcal{N} / N_{p}$ particles.

4. The procedure is iterated going back to item 1 .

We mentioned that the MCP method can be time consuming. We are attempting to improve the Monte Carlo integrations by trying variance reduction techniques, which build on the central limit theorem [22,24], and also by trying quasirandom sequences (also called lowdiscrepancy sequences) in place of pseudorandom sequences [24,25]. Quasirandom sequences allow one to break the "curse of dimensionality" in grid-based multidimensional integration, giving a true error bound (i.e., not probabilistic) of order $(\log \mathcal{N})^{k-1} / \mathcal{N}$, with only logarithmic dependence on the dimension $k$ of the space. We are also attempting to improve the Monte Carlo integrations by considering a discrete Fourier transform method for nonequidistant points, called nonequidistant fast Fourier transform method, which is employed by the subroutine library NFFT [26].

As an alternative to $\mathrm{MCP}$ we are investigating a scheme based on the standard PIC procedure of charge deposition to a grid, followed by additional filtering using wavelets. This has been applied in beam dynamics in Ref. [27]. We are also pursuing kernel density estimation methods from statistics $[28,29]$. These are closely related to quasiinterpolation methods from scattered data approximation [30-32].

\section{LINEAR INTEGRAL EQUATION TO DETERMINE THE GAIN FACTOR}

Recalling the definitions of force components in (28), we now consider the case when $F_{z 2}$ and $F_{x}$ are zero and $F_{z 1}$ can be approximated by an impedance model. Our beam frame equations of motion (29) become

$$
\zeta^{\prime}=A(s) \zeta+G(\zeta, s ; f)
$$

where the collective force term $G(\zeta, s ; f)$ in (49) is defined by

$$
\begin{gathered}
G(\zeta, s ; f)=\left[0, G_{2}(z, s ; f), 0,0\right]^{T}, \\
G_{2}(z, s ; f)=-\frac{r_{e}}{\gamma_{r}} \int_{\mathbb{R}^{4}} d \zeta^{\prime} W\left(z-z^{\prime}, s\right) f\left(\zeta^{\prime}, s\right), \\
r_{e}=\frac{Z_{0} q^{2}}{4 \pi m c},
\end{gathered}
$$

and where $f$ is the beam frame phase space density in (31). The radiation wake function $W$ and the radiation impedance $Z$ form a Fourier transform pair:

$$
\begin{aligned}
& Z(k, s)=\int_{\mathbb{R}} d z W(z, s) \exp (-i k z), \\
& W(z, s)=\frac{1}{2 \pi} \int_{\mathbb{R}} d k Z(k, s) \exp (i k z),
\end{aligned}
$$

and we have $Z(0, s)=0$. The vector field defined by the right-hand side (rhs) of (49) is divergence free, thus the Vlasov equation is

$$
D_{2} f(\zeta, s)+D_{1} f(\zeta, s)[A(s) \zeta+G(\zeta, s ; f)]=0,
$$

where $D_{2} f$ is the partial derivative of $f$ with respect to (w.r.t.) $s$ and where $D_{1} f$ is the row vector consisting of the partial derivatives w.r.t. $z, p_{z}, x$, and $p_{x}$, respectively.

The longitudinal spatial density, $\rho$, is defined by (35) and its $z$-Fourier transform, $\tilde{\rho}$, by (2). Our goal is to characterize $\tilde{\rho}$ at wave numbers, $k$, corresponding to the feared microbunching instability. In terms of $\tilde{\rho}$ the collective force can be written as

$$
G_{2}(z, s ; f)=-\frac{r_{e}}{2 \pi \gamma_{r}} \int_{\mathbb{R}} d k Z(k, s) \tilde{\rho}(k, s) \exp (i k z) .
$$

We wish to study linear stability of a "smooth" solution $f_{0}$ of the initial value problem

$$
\begin{gathered}
D_{2} f_{0}(\zeta, s)+D_{1} f_{0}(\zeta, s)\left[A(s) \zeta+G\left(\zeta, s ; f_{0}\right)\right]=0, \\
f_{0}(\zeta, 0)=a_{0}(\zeta)
\end{gathered}
$$

That is, we write

$$
f=f_{0}+f_{1},
$$

linearize in $f_{1}$, and then look for growth (in some appropriate sense) of an initial value of $f_{1}$. Here the spatial density from $f_{1}$ will contain wavelengths less than those of any appreciable component of $f_{0}$; that is the meaning of smooth as an attribute of $f_{0}$ depending on the choice of $f_{1}$. We emphasize that $f_{0}$ is smooth relative to $f_{1}$, not necessarily smooth by some absolute standard.

Of course it is difficult to find an $f_{0}$ satisfying (55) for an entirely arbitrary initial value $a_{0}$; to do so would be the same as solving the full problem, thus obviating any reason to linearize. There may be some $a_{0}$, however, for which the collective force $G_{2}\left(z, s ; f_{0}\right)$ is initially zero and remains so; in that case we can solve (55) and (56) by characteristics. Defining

$$
\begin{aligned}
\tilde{\rho}_{i}(k, s) & =\int_{\mathbb{R}} d z \rho_{i}(z, s) \exp (-i k z), \\
\rho_{i}(z, s) & =\int_{\mathbb{R}^{3}} d p_{z} d x d p_{x} f_{i}(\zeta, s), \quad(i=0,1),
\end{aligned}
$$

we observe that the force will be initially zero if the Fourier spectrum of $\rho_{0}(\cdot, 0)$ is zero within the support of the impedance: $Z(k, 0) \tilde{\rho}_{0}(k, 0)=0$. This is the case for a coasting beam but could also arise with a bunched beam through shielding of CSR by the vacuum chamber, so that $Z$ is essentially zero at wavelengths longer than the shielding threshold $\lambda_{0}$. By coasting beam we mean that $\rho_{0}(z, s)$ is independent of $z$ for each $s$.

If the coasting beam condition holds initially then, as shown in Sec. III C, it holds for all $s$ if the energy chirp is linear. Furthermore the bunched beam under shielding could also keep its spectrum below $k_{0}$ if bunch compres- 
sion were not too extreme. Thus we have at least two cases in which a solution of (55) and (56) is available. Others might be obtained by time domain numerical integration of (55) from a smooth initial condition, provided that very small wavelengths do not appear as the integration proceeds.

We proceed to linearize (53) about an arbitrary $f_{0}$, therefore allowing the case of a bunched beam, and cast the linearized equation into the form of the 2D integral equation (78) for $\tilde{\rho}_{1}$. The $2 \mathrm{D}$ equation for $\tilde{\rho}_{1}$ reduces to a $1 \mathrm{D}$ equation when $f_{0}$ models a coasting beam with linear energy chirp. In the case of the coasting beam in Sec. III C this $1 \mathrm{D}$ integral equation reduces to the results in $[12,13]$ since these authors deal with this model. Inserting (57) into (53) and applying (55) we obtain

$$
\begin{aligned}
& D_{2} f_{1}+D_{1} f_{1}\left[A(s) \zeta+G\left(\zeta, s ; f_{0}\right)\right] \\
& \quad=-D_{1} f_{0} G\left(\zeta, s ; f_{1}\right)-D_{1} f_{1} G\left(\zeta, s ; f_{1}\right) .
\end{aligned}
$$

Linearizing (59) in $f_{1}$ gives the following initial value problem for $f_{1}$ :

$$
\begin{gathered}
D_{2} f_{1}(\zeta, s)+D_{1} f_{1}(\zeta, s)\left[A(s) \zeta+G\left(\zeta, s ; f_{0}\right)\right] \\
=-D_{1} f_{0}(\zeta, s) G\left(\zeta, s ; f_{1}\right), \\
f_{1}(\zeta, 0)=a_{1}(\zeta) .
\end{gathered}
$$

Equation (60) is a linear first-order partial differentialintegral equation in the independent variables $\zeta, s$ for the $s$ evolution of $f_{1}$. The initial condition (61) will contain the perturbation involving wavelengths of interest.

\section{A. Derivation of $2 D$ integral equation for $\tilde{\rho}_{1}$}

We now proceed to derive from (60) and (61) the integral equation (78) for the Fourier transform $\tilde{\rho}_{1}$ of $\rho_{1}$. From a solution of this integral equation we could obtain the full perturbation $f_{1}$ by solving (60) using the method of characteristics. This follows from the fact that $G_{2}\left(z, s ; f_{1}\right)$ in (54) depends on $f_{1}$ only via $\tilde{\rho}_{1}$, which in turn follows from the 1D treatment of the collective force.

To convert (60) and (61) to an integral equation we need the characteristic equation for (55) which is

$$
\zeta^{\prime}=A(s) \zeta+G\left(\zeta, s ; f_{0}\right)
$$

and we need two general properties of its solutions. We write the general solution of (62) as

$$
\zeta=\varphi\left(s, s_{0}, \zeta_{0}\right)
$$

where $\varphi\left(s_{0}, s_{0}, \zeta_{0}\right)=\zeta_{0}$. The basic property of $\varphi$ is

$$
\varphi\left[s_{2}, s_{1}, \varphi\left(s_{1}, s_{0}, \zeta\right)\right]=\varphi\left(s_{2}, s_{0}, \zeta\right) .
$$

We assume solutions exist uniquely. We also invoke a notation for the Jacobian matrix,

$$
\Psi\left(s, s_{0}, \zeta_{0}\right)=D_{3} \varphi\left(s, s_{0}, \zeta_{0}\right),
$$

where $D_{3} \varphi\left(s, s_{0}, \zeta_{0}\right)$ is the $4 \times 4$ Jacobian matrix whose $i$ th column consists of the partial derivatives of $\varphi$ w.r.t. the $i$ th component of $\zeta_{0}$. Since the rhs of (62) is divergence free, $\operatorname{det}(\Psi)=1$. Differentiating the identity $\zeta=$ $\varphi\left[s, s_{0}, \varphi\left(s_{0}, s, \zeta\right)\right]$, obtained from (64), with respect to $\zeta$, the matrix inverse of $\Psi\left(s, s_{0}, \zeta_{0}\right)$ is

$$
\Psi^{-1}\left(s, s_{0}, \zeta_{0}\right)=\Psi\left[s_{0}, s, \varphi\left(s, s_{0}, \zeta_{0}\right)\right] .
$$

We can now write the solution of Eqs. (55) and (56) in terms of $\varphi$ as

$$
f_{0}(\zeta, s)=a_{0}[\varphi(0, s, \zeta)] .
$$

Solving (63) for $\zeta_{0}$ gives $\zeta_{0}=\varphi\left(s_{0}, s, \zeta\right)$ and, for $s_{0}=0$, $\zeta_{0}=\varphi(0, s, \zeta)$ whence

$$
a_{0}\left(\zeta_{0}\right)=f_{0}\left[\varphi\left(s, 0, \zeta_{0}\right), s\right] .
$$

Equation (55) is the differential statement that $f_{0}$ is constant along characteristics, whereas (68) states this more explicitly in terms of the characteristics and the values of $f_{0}$ on the characteristics.

We now examine the evolution of $f_{1}$ along the characteristics of the unperturbed problem. Thus we define $g$ by

$$
g(\zeta, s)=f_{1}[\varphi(s, 0, \zeta), s],
$$

and this gives, by (60) and the definition of $\varphi$,

$$
\begin{aligned}
D_{2} g(\zeta, s)= & D_{1} f_{1}[\varphi(s, 0, \zeta), s] D_{1} \varphi(s, 0, \zeta) \\
& +D_{2} f_{1}[\varphi(s, 0, \zeta), s] \\
= & -\left\{D_{1} f_{0}[\varphi(s, 0, \zeta), s]\right\}_{2} G_{2}\left[\varphi_{1}(s, 0, \zeta), s ; f_{1}\right],
\end{aligned}
$$

$$
g(\zeta, 0)=f_{1}(\zeta, 0)=a_{1}(\zeta),
$$

where the column vector $D_{1} \varphi(s, 0, \zeta)$ is the derivative of $\varphi(s, 0, \zeta)$ with respect to $s$. The replacement of $f_{1}$ by $g$ in (69) is often called "passing to the interaction picture," since the evolution of $g$ is governed just by the collective force, the "interaction."

By differentiating (68) we have

$$
D a_{0}(\zeta)=D_{1} f_{0}[\varphi(s, 0, \zeta), s] D_{3} \varphi(s, 0, \zeta),
$$

and hence by (65) and (66)

$$
D_{1} f_{0}[\varphi(s, 0, \zeta), s]=D a_{0}(\zeta) \Psi[0, s, \varphi(s, 0, \zeta)],
$$

where $D a_{0}(\zeta)$ is the row vector consisting of the partial derivatives of $a_{0}$ w.r.t. $z, p_{z}, x$, and $p_{x}$. From (73) we see that the first factor on the right-hand side of (70) can be written

$$
\left\{D_{1} f_{0}[\varphi(s, 0, \zeta), s]\right\}_{2}=D a_{0}(\zeta) \Psi_{* 2}[0, s, \varphi(s, 0, \zeta)] .
$$

Let $V$ be a matrix then $V_{* j}$ and $V_{i *}$ denoted the $j$ th column and $i$ th row of $V$, respectively. The symbol $V_{i *}^{T}$ will denote the transpose of $V_{i^{*}}$ (not the $i$ th row of $V^{T}$ ). In view of (54) and (74), the equation (70) for $g$ takes the form 


$$
\begin{aligned}
D_{2} g(\zeta, s)= & \frac{r_{e}}{2 \pi \gamma_{r}} D a_{0}(\zeta) \Psi_{* 2}[0, s, \varphi(s, 0, \zeta)] \\
& \times \int_{\mathbb{R}} d k Z(k, s) \tilde{\rho}_{1}(k, s) \exp \left[i k \varphi_{1}(s, 0, \zeta)\right]
\end{aligned}
$$

From (58) and (69) we obtain

$$
\begin{aligned}
\tilde{\rho}_{1}(k, s) & =\int_{\mathbb{R}^{4}} d \zeta f_{1}(\zeta, s) \exp (-i k z) \\
& =\int_{\mathbb{R}^{4}} d \zeta g(\zeta, s) \exp \left[-i k \varphi_{1}(s, 0, \zeta)\right] .
\end{aligned}
$$

We have used the fact that $\operatorname{det}(\Psi)=1$ as mentioned after (65). It is now easy to turn (75) into an integral equation for $\tilde{\rho}_{1}$. First, integrating (75) over $s$ and using (71) gives

$$
\begin{aligned}
g(\zeta, s)= & a_{1}(\zeta)+\frac{r_{e}}{2 \pi \gamma_{r}} \int_{0}^{s} d \tau \int_{\mathbb{R}} d k \tilde{\rho}_{1}(k, \tau) Z(k, \tau) \\
& \times D a_{0}(\zeta) \Psi_{* 2}[0, \tau, \varphi(\tau, 0, \zeta)] \exp \left[i k \varphi_{1}(\tau, 0, \zeta)\right]
\end{aligned}
$$

Then, integrating (77) over $\zeta$ after the multiplication by $\exp \left[-i k \varphi_{1}(s, 0, \zeta)\right]$ gives

$$
\begin{aligned}
\tilde{\rho}_{1}(k, s)= & \hat{a}_{1}(k, s) \\
& +\int_{0}^{s} d \tau \int_{\mathbb{R}} d k_{1} \mathcal{K}\left(s, \tau, k, k_{1}\right) \tilde{\rho}_{1}\left(k_{1}, \tau\right),
\end{aligned}
$$

where

$$
\begin{aligned}
\mathcal{K}\left(s, \tau, k, k_{1}\right)= & \frac{r_{e}}{2 \pi \gamma_{r}} Z\left(k_{1}, \tau\right) \\
& \times \int_{\mathbb{R}^{4}} d \zeta D a_{0}(\zeta) \Psi_{* 2}[0, \tau, \varphi(\tau, 0, \zeta)] \\
& \times \exp \left\{-i\left[k \varphi_{1}(s, 0, \zeta)-k_{1} \varphi_{1}(\tau, 0, \zeta)\right]\right\}
\end{aligned}
$$

$$
\hat{a}_{1}(k, s)=\int_{\mathbb{R}^{4}} d \zeta a_{1}(\zeta) \exp \left[-i k \varphi_{1}(s, 0, \zeta)\right]
$$

Thus our 2D integral equation is (78) with the kernel given in (79) and the nonhomogeneous term in (80).

\section{B. 2D integral equation for $\tilde{\rho}_{1}$ when $G\left(\zeta, s ; f_{0}\right)=0$}

Equations (79) and (80) both simplify and become more explicit when

$$
G\left(\zeta, s ; f_{0}\right)=0
$$

which includes the coasting beam case since $Z(0, s)=0$. From (81) it follows that $\varphi$ and $\Psi$ do not depend on $f_{0}$ and are given by

$$
\varphi(s, \tau, \zeta)=\Phi(s, \tau) \zeta, \quad \Psi(s, \tau, \zeta)=\Phi(s, \tau)
$$

where the $4 \times 4$ matrix function $\Phi(s, \tau)$ is the unique solution of

$$
D_{1} \Phi(s, \tau)=A(s) \Phi(s, \tau), \quad \Phi(\tau, \tau)=I_{4 \times 4} .
$$

Since $\operatorname{Tr}[A(s)]=0$ and $\Phi(\tau, \tau)=I_{4 \times 4}$, we have $\operatorname{det}[\Phi(s, \tau)]=1$ which is consistent with the more general case from Sec. III A. The transfer map (principal solution matrix) $\Phi(s, \tau)$ can be written in terms of the dispersion function,

$$
D(s, \tau)=\int_{\tau}^{s} d s^{\prime} \int_{\tau}^{s^{\prime}} d s^{\prime \prime} \kappa\left(s^{\prime \prime}\right)
$$

and the momentum compaction function

$$
R_{56}(s, \tau)=-\int_{\tau}^{s} d s^{\prime} \kappa\left(s^{\prime}\right) D\left(s^{\prime}, \tau\right)
$$

as

$$
\Phi(s, \tau)=\left(\begin{array}{cccc}
1 & R_{56}(s, \tau) & -D^{\prime}(s, \tau) & D(s, \tau)-(s-\tau) D^{\prime}(s, \tau) \\
0 & 1 & 0 & 0 \\
0 & D(s, \tau) & 1 & s-\tau \\
0 & D^{\prime}(s, \tau) & 0 & 1
\end{array}\right)
$$

where $D^{\prime}(s, \tau)=D_{1} D(s, \tau)$. The inverse is $\Phi^{-1}(s, \tau)=$ $\Phi(\tau, s)$. Using (82), Eq. (67) becomes

$$
f_{0}(\zeta, s)=a_{0}(\Phi(0, s) \zeta)
$$

In order to derive the new forms of $\mathcal{K}$ and $\hat{a}_{1}$, we need the 4D Fourier transforms of $a_{0}$ and $a_{1}, \tilde{a}_{i}(\kappa)=$ $\int_{\mathbb{R}^{4}} d \zeta a_{i}(\zeta) \exp \left(-i \kappa^{T} \zeta\right) \quad$ where $i=0,1$ and $\kappa=$ $\left(\kappa_{1}, \kappa_{2}, \kappa_{3}, \kappa_{4}\right)^{T}$. Since $\Phi_{* 2}[0, \tau, \varphi(\tau, 0, \zeta)]=\Phi_{* 2}(0, \tau)$, $\varphi_{1}(s, 0, \zeta)=\Phi_{1 *}(s, 0) \zeta$ and $\int_{\mathbb{R}^{4}} d \zeta D a_{0}(\zeta) \exp \left(-i \kappa^{T} \zeta\right)=$ $i \tilde{a}_{0}(\kappa) \kappa^{T}$, the kernel $\mathcal{K}$ is replaced by $\mathcal{K}_{1}$, where

$$
\begin{aligned}
\mathcal{K}_{1}\left(s, \tau, k, k_{1}\right)= & \frac{i r_{e}}{2 \pi \gamma_{r}} Z\left(k_{1}, \tau\right) \tilde{a}_{0}\left[k \Phi_{1 *}^{T}(s, 0)\right. \\
& \left.-k_{1} \Phi_{1 *}^{T}(\tau, 0)\right] k \Phi_{12}(s, \tau) .
\end{aligned}
$$

Here we used the fact that $\left[k \Phi_{1 *}(s, 0)-\right.$ $\left.k_{1} \Phi_{1 *}(\tau, 0)\right] \Phi_{* 2}(0, \tau)=k \Phi_{12}(s, \tau)$. It is easy to check that $\hat{a}_{1}(k, s)=\tilde{a}_{1}\left[k \Phi_{1 *}^{T}(s, 0)\right]$, whence (78) reads as

$$
\begin{aligned}
\tilde{\rho}_{1}(k, s)= & \tilde{a}_{1}\left[k \Phi_{1 *}^{T}(s, 0)\right] \\
& +\int_{0}^{s} d \tau \int_{\mathbb{R}} d k_{1} \mathcal{K}_{1}\left(s, \tau, k, k_{1}\right) \tilde{\rho}_{1}\left(k_{1}, \tau\right) .
\end{aligned}
$$


If we choose (as we do in our two models) $a_{0}$ and $a_{1}$ to be of the form

$$
\begin{gathered}
a_{0}(\zeta)=\mu(z) a_{C B}\left(p_{z}-h z, x, p_{x}\right), \\
a_{1}(\zeta)=\varepsilon(z) a_{0}(\zeta),
\end{gathered}
$$

where $a_{C B}$ is normalized then

$$
\tilde{a}_{0}(\kappa)=\tilde{\mu}\left(\kappa_{1}+h \kappa_{2}\right) \tilde{a}_{C B}\left(\kappa_{2}, \kappa_{3}, \kappa_{4}\right) .
$$

Here $\tilde{\mu}$ and $\tilde{a}_{C B}$ are the $1 \mathrm{D}$ and 3D Fourier transforms, respectively. Note that the chirp parameter $h$, which quantifies the linear energy chirp, is the "slope" of the correlation. With (90) the integral equation (89) gets further structure since by (92) we have

$$
\begin{aligned}
& \tilde{a}_{0}\left[k \Phi_{1 *}^{T}(s, 0)-k_{1} \Phi_{1 *}^{T}(\tau, 0)\right] \\
& \quad=\tilde{\mu}\left(\frac{k}{C(s)}-\frac{k_{1}}{C(\tau)}\right) \tilde{a}_{C B}\left[k d(s)-k_{1} d(\tau)\right],
\end{aligned}
$$

where

$$
\begin{gathered}
d(s)=\left[\Phi_{12}(s, 0), \Phi_{13}(s, 0), \Phi_{14}(s, 0)\right]^{T}, \\
C(s)=\left[1+h R_{56}(s)\right]^{-1} \\
R_{56}(s)=R_{56}(s, 0)=\Phi_{12}(s, 0) .
\end{gathered}
$$

We assume that $C(s)>0$ for $s \in\left[0, s_{f}\right]$. Since $C(s)$ is the compression factor at $s$, this condition is always fulfilled in a chicane. Inserting (93) into (88), $\mathcal{K}_{1}$ becomes

$$
\begin{aligned}
\mathcal{K}_{2}\left(s, \tau, k, k_{1}\right)= & \frac{i r_{e}}{2 \pi \gamma_{r}} Z\left(k_{1}, \tau\right) \tilde{\mu}\left(\frac{k}{C(s)}-\frac{k_{1}}{C(\tau)}\right) \\
& \times \tilde{a}_{C B}\left[k d(s)-k_{1} d(\tau)\right] k \Phi_{12}(s, \tau),
\end{aligned}
$$

whence (89) reads as

$$
\begin{aligned}
\tilde{\rho}_{1}(k, s)= & \tilde{a}_{1}\left[k \Phi_{1 *}^{T}(s, 0)\right] \\
& +\int_{0}^{s} d \tau \int_{\mathbb{R}} d k_{1} \mathcal{K}_{2}\left(s, \tau, k, k_{1}\right) \tilde{\rho}_{1}\left(k_{1}, \tau\right) .
\end{aligned}
$$

Note that for both models, used for the numerical computations presented in Sec. IV, $\varepsilon$ is given by (129) where $k_{0}>0$ is the wave number of the initial modulation. Using (129),

$$
\begin{aligned}
\tilde{a}_{1}(\kappa)= & \frac{A}{2}\left[\tilde{\mu}\left(\kappa_{1}+h \kappa_{2}-k_{0}\right)+\tilde{\mu}\left(\kappa_{1}+h \kappa_{2}+k_{0}\right)\right] \\
& \times \tilde{a}_{C B}\left(\kappa_{2}, \kappa_{3}, \kappa_{4}\right),
\end{aligned}
$$

and thus

$$
\begin{aligned}
\tilde{a}_{1}\left[k \Phi_{1 *}^{T}(s, 0)\right]= & \frac{A}{2}\left[\tilde{\mu}\left(\frac{k}{C(s)}-k_{0}\right)+\tilde{\mu}\left(\frac{k}{C(s)}+k_{0}\right)\right] \\
& \times \tilde{a}_{C B}[k d(s)] .
\end{aligned}
$$

\section{Coasting beam case and 1D integral equation}

The simplest case which satisfies (81) is where $a_{0}$ is given by (90) with $\mu(z)=\mu_{c}$ a constant. Thus with (91) we have

$$
\begin{gathered}
a_{0}(\zeta)=\mu_{c} a_{C B}\left(p_{z}-h z, x, p_{x}\right), \\
a_{1}(\zeta)=\varepsilon(z) a_{0}(\zeta) .
\end{gathered}
$$

Note that in this case $a_{0}$ is not normalizable. Equation (81) now follows from the fact that

$$
\rho_{0}(z, s)=\int_{\mathbb{R}^{3}} d \zeta_{r} f_{0}\left[\Phi(0, s)\left(z, \zeta_{r}\right)^{T}, 0\right]
$$

is independent of $z$, where $\zeta_{r}=\left(p_{z}, x, p_{x}\right)^{T}$ which we now show.

We can write

$$
\Phi(s, 0)=\left(\begin{array}{cc}
1 & d^{T}(s) \\
0 & \Phi_{r}(s)
\end{array}\right), \quad \Phi(0, s)=\left(\begin{array}{cc}
1 & e(s) \\
0 & \Phi_{r}^{-1}(s)
\end{array}\right)
$$

where $e(s)=\left[-R_{56}(s), D^{\prime}(s),-D(s)\right]$. We make the change of variables $\zeta_{r} \rightarrow \hat{\zeta}_{r}=\left(\hat{p}_{z}, \hat{x}, \hat{p}_{x}\right)^{T}=\Phi_{r}^{-1}(s) \zeta_{r}$. Since $\operatorname{det}\left[\Phi_{r}(s)\right]=1$,

$$
\begin{aligned}
\Phi(0, s)\left(z, \zeta_{r}\right)^{T} & =\Phi(0, s)\left(\begin{array}{c}
z \\
\Phi_{r}(s) \hat{\zeta}_{r}
\end{array}\right) \\
& =\left(\begin{array}{c}
z+e(s) \Phi_{r}(s) \hat{\zeta}_{r} \\
\hat{\zeta}_{r}
\end{array}\right),
\end{aligned}
$$

and $f_{0}\left(z, \zeta_{r}, 0\right)=\mu_{c} a_{C B}\left(p_{z}-h z, x, p_{x}\right)$ we obtain

$$
\begin{aligned}
\rho_{0}(z, s)= & \mu_{c} \int_{\mathbb{R}^{3}} d \hat{p}_{z} d \hat{x} d \hat{p}_{x} a_{C B}\left(\left[1+h R_{56}(s)\right] \hat{p}_{z}\right. \\
& \left.-h\left\{z+D^{\prime}(s) \hat{x}+\left[s D^{\prime}(s)-D(s)\right] \hat{p}_{x}\right\}, \hat{x}, \hat{p}_{x}\right) \\
= & \mu_{c}\left[1+h R_{56}(s)\right]^{-1}=\mu_{c} C(s),
\end{aligned}
$$

which is also in [13].

Having thus shown that (100) implies (81), we can now apply the results of Sec. III B. Taking the 4D Fourier transform of (100) and (101) we obtain

$$
\begin{gathered}
\tilde{a}_{0}(\kappa)=2 \pi \mu_{c} \delta\left(\kappa_{1}+h \kappa_{2}\right) \tilde{a}_{C B}\left(\kappa_{2}, \kappa_{3}, \kappa_{4}\right), \\
\tilde{a}_{1}(\kappa)=\tilde{\varepsilon}\left(\kappa_{1}+h \kappa_{2}\right) \mu_{c} \tilde{a}_{C B}\left(\kappa_{2}, \kappa_{3}, \kappa_{4}\right) .
\end{gathered}
$$

It follows that

$$
\tilde{\mu}(k)=2 \pi \mu_{c} \delta(k)
$$

$$
\tilde{a}_{1}\left[k \Phi_{1 *}^{T}(s, 0)\right]=\tilde{\varepsilon}[k / C(s)] \mu_{c} \tilde{a}_{C B}[k d(s)] .
$$

Because of the $\delta$ function in (108), the $k_{1}$ integration in (97) can be performed and we have 


$$
\begin{aligned}
\tilde{\rho}_{1}(k, s)= & \tilde{\varepsilon}[k / C(s)] \mu_{c} \tilde{a}_{C B}[k d(s)] \\
& +\int_{0}^{s} d \tau \hat{\mathcal{K}}_{C B}(s, \tau, k) \tilde{\rho}_{1}\left(\frac{k C(\tau)}{C(s)}, \tau\right),
\end{aligned}
$$

where

$$
\begin{aligned}
\hat{\mathcal{K}}_{C B}(s, \tau, k)= & \frac{i k r_{e} \mu_{c}}{\gamma_{r}} C(\tau) \Phi_{12}(s, \tau) Z\left(\frac{k C(\tau)}{C(s)}, \tau\right) \\
& \times \tilde{a}_{C B}\left(k d(s)-\frac{k C(\tau)}{C(s)} d(\tau)\right) .
\end{aligned}
$$

Replacing $k$ in (110) by $k C(s)$ we obtain

$$
\begin{aligned}
\tilde{\rho}_{1}[k C(s), s]= & \tilde{\varepsilon}(k) \mu_{c} \tilde{a}_{C B}[k C(s) d(s)] \\
& +\int_{0}^{s} d \tau \mathcal{K}_{C B}(s, \tau, k) \tilde{\rho}_{1}(k C(\tau), \tau),
\end{aligned}
$$

where

$$
\begin{aligned}
\mathcal{K}_{C B}(s, \tau, k)= & \hat{\mathcal{K}}_{C B}[s, \tau, k C(s)] \\
= & \frac{i k r_{e} \mu_{c}}{\gamma_{r}} C(s) C(\tau) \Phi_{12}(s, \tau) Z[k C(\tau), \tau] \\
& \times \tilde{a}_{C B}[k C(s) d(s)-k C(\tau) d(\tau)] .
\end{aligned}
$$

Because of (105) we have $\tilde{\rho}_{0}[k C(s), s]=2 \pi \mu_{c} \delta(k)$ whence

$$
\tilde{\rho}_{1}(k, s)=\tilde{\rho}(k, s), \quad \text { for } k \neq 0 .
$$

Thus by (112), for $k \neq 0$,

$$
\begin{aligned}
\tilde{\rho}[k C(s), s]= & \tilde{\varepsilon}(k) \mu_{c} \tilde{a}_{C B}[k C(s) d(s)] \\
& +\int_{0}^{s} d \tau \mathcal{K}_{C B}(s, \tau, k) \tilde{\rho}[k C(\tau), \tau] .
\end{aligned}
$$

We now compare with $[12,13]$. In the special case of the analytical model we have the $\varepsilon$ of (129) and the $a_{C B}$ of (137) where $\rho_{c}, \rho_{t}$ are given by (127) and (128), respectively. It is thus easy to show that, for the analytical model, (112) is Eq. (30) in [13] where (as in case of our numerical computation-see Sec. IV) $\alpha_{0}=0$. Thus (112) is the generalization of Eq. (30) in [13] to general $\varepsilon, a_{C B}, \alpha_{0}$. The relation to [13] is based on the equality $g_{k}(s)=$ $\tilde{\rho}_{1}[k C(s), s]$. Furthermore, in the special case of the analytical model it is easy to show that (115) is Eq. (20) in [12] when $k$ is taken $k_{0}$ in (115). The relation to [12] is based on the equality $b[k(s) ; s]=\tilde{\rho}\left[k_{0} C(s), s\right]$.

Solving (112) for every value of the parameter $k$, one would obtain the function $\tilde{\rho}_{1}$ whence, due to the remark at the beginning of Sec. III A, one then could derive the full perturbation $f_{1}$ of $f$ by solving (60) by the method of characteristics.

Defining the linear operator $Q$ and the functions $\beta$ and $\gamma$ by

$$
\begin{gathered}
(Q F)(k, s)=\int_{0}^{s} d \tau \mathcal{K}_{C B}(s, \tau, k) F(k, \tau), \\
\beta(k, s)=\tilde{\rho}[k C(s), s], \quad \gamma(k, s)=\mu_{c} \tilde{a}_{C B}[k C(s) d(s)],
\end{gathered}
$$

we can write (115) as

$$
\beta=\tilde{\varepsilon}(k) \gamma+Q \beta,
$$

where of course $k \neq 0$. In [12] an approximate solution of (117) is constructed by iteration:

$$
\beta^{(n+1)}=\tilde{\varepsilon}(k) \gamma+Q \beta^{(n)},
$$

where $\beta^{(n)}$ aims to approximate $\beta$ with increasing accuracy when $n$ increases. Using the fact that $Q \tilde{\varepsilon}(k) F=$ $\tilde{\varepsilon}(k) Q F$ and choosing $\beta^{(0)}=\tilde{\varepsilon}(k) \gamma$ we obtain for $n=2$

$$
\beta^{(2)}=\left(Q^{2}+Q+1\right) \beta^{(0)}=\tilde{\varepsilon}(k)\left(Q^{2}+Q+1\right) \gamma .
$$

The gain factor defined in (1) then reads as

$$
\text { (S) } \begin{aligned}
\left(k_{0}\right) & =\left|\frac{\tilde{\rho}\left[k_{0} C\left(s_{f}\right), s_{f}\right]}{\tilde{\rho}\left(k_{0}, 0\right)}\right| \approx\left|\frac{\beta\left(k_{0}, s_{f}\right)}{\beta\left(k_{0}, 0\right)}\right| \\
& \approx\left|\frac{\beta^{(2)}\left(k_{0}, s_{f}\right)}{\beta^{(2)}\left(k_{0}, 0\right)}\right| \\
& =\left|\frac{\tilde{\varepsilon}\left(k_{0}\right)\left[\left(Q^{2}+Q+1\right) \gamma\right]\left(k_{0}, s_{f}\right)}{\tilde{\varepsilon}\left(k_{0}\right)\left[\left(Q^{2}+Q+1\right) \gamma\right]\left(k_{0}, 0\right)}\right| \\
& =\left|\frac{\left[\left(Q^{2}+Q+1\right) \gamma\right]\left(k_{0}, s_{f}\right)}{\left[\left(Q^{2}+Q+1\right) \gamma\right]\left(k_{0}, 0\right)}\right| \\
& =\frac{1}{\mu_{c}}\left|\left[\left(Q^{2}+Q+1\right) \gamma\right]\left(k_{0}, s_{f}\right)\right|=: \operatorname{ss}^{(2)}\left(k_{0}\right),
\end{aligned}
$$

where we also used the fact that $k_{0} \neq 0$ and that $\gamma(k, 0)=$ $\mu_{c}$ (the latter follows from the normalization of $a_{C B}$ ). We call $\mathfrak{S S}^{(2)}\left(k_{0}\right)$ the analytical gain factor and it is used for the analytical model in Sec. IV and in [12]. Here the first approximation entails the approximations leading to (117). We are studying the nature of these approximations including the fact that in our two models $\varepsilon(z)=A \cos \left(k_{0} z\right)$ so that

$$
\tilde{\varepsilon}(k)=\pi A\left\{\delta\left(k-k_{0}\right)+\delta\left(k+k_{0}\right)\right\} .
$$

Here we are interested only in producing a rough figure of merit of instability. A numerical comparison of $\left(\mathfrak{S}\left(k_{0}\right)\right.$ in the MCP model with ${ }^{(5)}\left(k_{0}\right)$ in the analytical model is done in Sec. IV.

\section{Remarks on nonlinear chirp}

The assumption of linear chirp is usually not realistic, even though one tries to minimize nonlinearity in bunch compressor designs. Venturini [33] has raised the question of whether nonlinear terms in the chirp might affect the microbunching instability, having noticed a discrepancy 
between simulations with and without nonlinear terms (albeit simulations that differed in other respects as well). Approaching this question through the integral equation, we can choose in place of (100) an initial density of the form

$$
a_{0}(\zeta)=\mu_{c} a_{C B}\left[p_{z}-h \alpha(z), x, p_{x}\right] .
$$

That is, we still assume an initial coasting beam but with nonlinear chirp function $\alpha(z)$. For the special case of a cubic chirp, $\alpha(z)=z+b z^{3}$, which may be realistic in some cases, one can evaluate $\tilde{a}_{0}$ in terms of the Airy function [34]:

$$
\begin{gathered}
\tilde{a}_{0}(\kappa)=2 \pi \mu_{c}(3 v)^{-1 / 3} A i\left[ \pm(3 v)^{-1 / 3} u\right] \tilde{a}_{C B}\left(\kappa_{2} \cdot \kappa_{3}, \kappa_{4}\right), \\
u=\left|\kappa_{1}+h \kappa_{2}\right|, \quad v=\left|h b \kappa_{2}\right| .
\end{gathered}
$$

The + sign is chosen when $\kappa_{1}+h \kappa_{2}$ and $h b \kappa_{2}$ have the same sign, the - sign when their signs are opposite. Since (123) lacks the delta function of (106), the integral equation is now in 2D. This may be the most interesting case for a first study of the 2D equation. Generalizing the calculation of (118) and (119), the solution might be approximated by iteration. Note that the concept of gain should be generalized in this case, since the compression is no longer determined by $C(s)$.

Note that the initial coasting beam condition of uniform spatial density is not maintained when the chirp is nonlinear, even if the collective force is turned off, since $\alpha(z)$ acquires a nonlinear dependence on $p_{z}$ under unperturbed propagation in the lattice giving, instead of (105),

$\rho_{0}(z, s)=\mu_{c} \int_{\mathbb{R}^{3}} d \hat{\zeta}_{r} a_{C B}\left\{\hat{p}_{z}-h \alpha\left[z+e(s) \Phi_{r}(s) \hat{\zeta}_{r}\right], \hat{x}, \hat{p}_{x}\right\}$.

Thus the spatial density becomes nonuniform in $z$ through chirp alone, and $G\left(\zeta, s ; f_{0}\right) \neq 0$ for $s>0$. Perhaps $G\left(\zeta, s ; f_{0}\right)$ is nevertheless sufficiently small to be neglected when $b$ is small; this must be checked.

Recall that $\operatorname{Ai}(x)$ has exponential decrease for $x>0$, but oscillates for $x<0$, and has discontinuous slope at $x=0$. Consequently, the behavior of (123) near $u=0$ at small $v$ will be complicated and will require close attention in any numerical or analytic study.

\section{FERMI@ELETTRA BUNCH COMPRESSOR STUDIES}

Here we study the FERMI@Elettra first bunch compressor system by applying the MCP solver to the MCP model and, to a lesser extent, by applying the analytical model which was considered by $[12,13]$. This bunch compressor system was proposed as a benchmark for testing codes. The complete layout of the system is shown in Fig. 4. The system consists of a four-dipole chicane between rf cavities and quadrupoles. Here we limit our study to the chicane with parameters as listed in Table I. The results are ob-
TABLE I. Chicane and beam parameters.

\begin{tabular}{lccc}
\hline \hline Parameter & Symbol & Value & Unit \\
\hline Energy reference particle & $E_{r}$ & 233 & $\mathrm{MeV}$ \\
Peak current & $I$ & 120 & $\mathrm{~A}$ \\
Bunch charge & $Q$ & 1 & $\mathrm{nC}$ \\
Normalized emittance & $\epsilon_{x}=\gamma \epsilon_{0}$ & 1 & $\mu \mathrm{m}$ \\
Alpha function & $\alpha_{0}$ & 0 & \\
Beta function & $\beta_{0}$ & 10 & $\mathrm{~m}$ \\
Linear energy chirp & $h$ & -12.6 & $1 / \mathrm{m}$ \\
Uncorrelated energy spread & $\sigma_{E}$ & 2 & $\mathrm{KeV}$ \\
Momentum compaction at $s_{f}$ & $R_{f}=R_{56}\left(s_{f}\right)$ & 0.057 & $\mathrm{~m}$ \\
Radius of curvature & $r_{0}$ & 5 & $\mathrm{~m}$ \\
Magnetic length & $L_{b}$ & 0.5 & $\mathrm{~m}$ \\
Distance 1st-2nd, 3rd-4th bend & $L_{1}$ & 2.5 & $\mathrm{~m}$ \\
Distance 2nd-3rd bend & $L_{2}$ & 1 & $\mathrm{~m}$ \\
\hline \hline
\end{tabular}

tained in the free space case; i.e., neglecting shielding effects from the vacuum chamber. In our simulations we noticed that $\tau_{B}$ in (34) has a negligible effect therefore we ignored its contribution. The lengths $L_{1}, L_{2}$, and $L_{b}$ are in terms of the lab frame $Z$ variable, thus the total length of the chicane is $8 \mathrm{~m}$. The total arclength traversed by the reference particle is $s_{f}=8.029 \mathrm{~m}$ and the compression factor at $s_{f}$ is $C\left(s_{f}\right)=\left[1+h R_{56}\left(s_{f}\right)\right]^{-1}=3.545$.

To study the microbunching instability, we choose the initial beam frame phase space density to be

$$
f\left(z, p_{z}, x, p_{x}, 0\right)=[1+\varepsilon(z)] a_{0}\left(z, p_{z}, x, p_{x}\right),
$$

where

$$
\begin{gathered}
a_{0}\left(z, p_{z}, x, p_{x}\right)=\mu(z) \rho_{c}\left(p_{z}-h z\right) \rho_{t}\left(x, p_{x}\right), \\
\rho_{c}\left(p_{z}\right)=\exp \left[-p_{z}^{2} / 2 \sigma_{u}^{2}\right] / \sqrt{2 \pi} \sigma_{u} \\
\rho_{t}\left(x, p_{x}\right)=\exp \left\{-\left[x^{2}+\left(\alpha_{0} x+\beta_{0} p_{x}\right)^{2}\right] / 2 \epsilon_{0} \beta_{0}\right\} / 2 \pi \epsilon_{0}, \\
\varepsilon(z)=A \cos \left(2 \pi z / \lambda_{0}\right)=A \cos \left(k_{0} z\right) .
\end{gathered}
$$

For the MCP model we use

$$
\mu(z)=\frac{\alpha}{4 a}\{\tanh [(z+a) / b]-\tanh [(z-a) / b]\},
$$

which is a smooth flattop density, even in $z$, with maximum at $z=0$. Thus the smooth $a_{0}$ is perturbed by a modulation, $\varepsilon$, with wavelength $\lambda_{0}$ and small amplitude $A$. For the analytical model we use

$$
\mu(z)=\mu_{c}=\text { const. }
$$

In the calculations we vary $\lambda_{0}$ and take $A=0.05, a=$ $1180 \mu \mathrm{m}$, and $b=150 \mu \mathrm{m}$. The purpose of $\alpha$ is to normalize $f$ in the MCP model, as demanded by (32). However, since it is a good approximation, we use $\alpha=1$ in our computations. Of course $f$ is not normalized in the analytical model. 


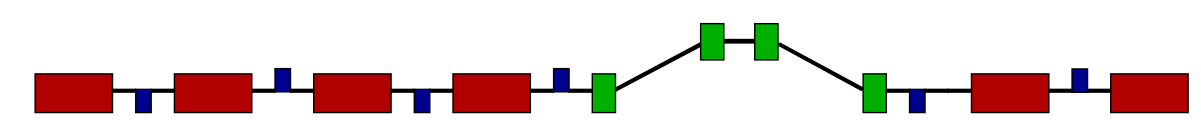

FIG. 4. (Color) Proposed layout of FERMI@Elettra first bunch compressor system. Accelerating rf cavities in red, quadrupole magnets in blue, drift sections in black, and dipoles in green. Chicane parameters are discussed in Table I.

The density $\rho_{c}\left(p_{z}-h z\right)$ contains the linear energy chirp which is created by off-crest rf acceleration such that particles in front of the reference particle gain less energy than particles behind the reference particle. This creates the correlation needed for bunch compression. The uncorrelated energy spread $\sigma_{E}=2 \mathrm{KeV}$ gives $\sigma_{u}=\sigma_{E} / E_{r}=$ $8.6 \times 10^{-6}$.

Taking the limit $b \rightarrow 0+$ in (130) we get $\mu \rightarrow \mu_{0}$ where $\mu_{0}(z)=(\alpha / 2 a) I_{(-a, a)}(z)$ and $I_{(-a, a)}$ is the indicator function of the open interval $(-a, a)$. The Fourier transform of $\mu_{0}$ is $\tilde{\mu}_{0}(k)=\alpha \sin (k a) / k a=: \alpha \operatorname{sinc}(k a)$. The function $\mu_{0}$ is a rough pointwise approximation to $\mu$, so that the bunch length is $\approx 2 a$. We use the smooth $\mu$ instead of $\mu_{0}$ because the discontinuous $\mu_{0}$ gives rise to a Gibbs phenomenon which causes problems in our numerics.

To better understand the effect of the self-fields on $\tilde{\rho}$, we first discuss $\tilde{\rho}$ without the self-fields. The initial longitudinal spatial density is

$$
\rho(z, 0)=[1+\varepsilon(z)] \mu(z)
$$

whence $f\left(z, p_{z}, x, p_{x}, 0\right)=\rho(z, 0) \rho_{c}\left(p_{z}-h z\right) \rho_{t}\left(x, p_{x}\right)$. Let $C\left(s_{f}\right)=C_{f}$ and $R_{56}\left(s_{f}\right)=R_{f}$ then, without self-fields, the longitudinal spatial density at $s=s_{f}$ is

$$
\begin{aligned}
\rho\left(z, s_{f}\right) & =\int_{\mathbb{R}^{3}} d p_{z} d x d p_{x} f\left[\Phi\left(0, s_{f}\right) \zeta, 0\right] \\
& =\int_{\mathbb{R}^{3}} d p_{z} d x d p_{x} f\left(z-R_{f} p_{z}, p_{z}, x-s_{f} p_{x}, p_{x}, 0\right) \\
& =\int_{\mathbb{R}} d p_{z} \rho\left(z-R_{f} p_{z}, 0\right) \rho_{c}\left(p_{z} / C_{f}-h z\right) \\
& =C_{f} \int_{\mathbb{R}} d y \rho\left[C_{f}\left(z-R_{f} y\right), 0\right] \rho_{c}(y),
\end{aligned}
$$

where in the second equality we used the fact that $D\left(0, s_{f}\right)=0=D^{\prime}\left(0, s_{f}\right)$. It is easy to check that $\rho\left(\cdot, s_{f}\right)$ in (133) is even and so its first moment is zero. Note that (132) and (133) hold for both choices of $\mu$ (130) and (131). Returning to the choice (130), a short calculation shows that the second moment of $\rho\left(\cdot, s_{f}\right)$ is equal to $1 / C_{f}^{2}$ times the second moment of $\rho(\cdot, 0)$ plus the term $R_{f}^{2} \sigma_{u}^{2}$. The twoterm asymptotic expansion of (133) for small $\sigma_{u}$ is

$$
\begin{aligned}
\rho\left(z, s_{f}\right) \sim & C_{f}\left[\rho\left(C_{f} z, 0\right)+D_{1}^{2} \rho\left(C_{f} z, 0\right)\left(C_{f} R_{f} \sigma_{u}\right)^{2} / 2\right], \\
& \text { as } \sigma_{u} \rightarrow 0 .
\end{aligned}
$$

For our parameters, $\left(C_{f} R_{f} \sigma_{u}\right)^{2}=O\left(10^{-12} \mathrm{~m}^{2}\right)$ and, to very good approximation,

$$
\rho\left(z, s_{f}\right)=C_{f} \rho\left(C_{f} z, 0\right) .
$$

This is just (133) with $\rho_{c}$ replaced by the delta function (see also the discussion of Fig. 13). The approximation of (133) clearly shows the compression and the meaning of the term "compression factor" [see also Fig. 13 (left)]. The Fourier transform of (133) gives

$$
\tilde{\rho}\left(k, s_{f}\right)=\tilde{\rho}_{c}\left(k R_{f}\right) \tilde{\rho}\left(k / C_{f}, 0\right)
$$

where $\tilde{\rho}_{c}(k)=\exp \left[-k^{2} \sigma_{u}^{2} / 2\right]$. Thus, neglecting selffields, the compression effect in (136) is exact whereas in (135) it is approximate (but nearly exact).

As discussed in Sec. IIC we accurately calculate the evolution of the two-dimensional spatial density given the initial beam frame phase space density of the MCP model. We will present results for the spatial density but our main focus will be on the integrated quantities: the longitudinal spatial density $\rho(z, s)$ and the modulus of its Fourier transform $\tilde{\rho}(k, s)$. In particular, we calculate the gain factor (S) $\left(k_{0}\right)=\left|\tilde{\rho}\left(k_{0} C_{f}, s_{f}\right) / \tilde{\rho}\left(k_{0}, 0\right)\right|$ of (1) for initial modulation wavelengths $\lambda_{0}=2 \pi / k_{0} \geq 80 \mu \mathrm{m}$.

Figure 5 (left) shows the initial spatial density which has been generated by Monte Carlo sampling in the grid coordinates $\left(x_{1}, x_{2}\right)$ from Sec. II C. We note that there is no visible noise. We chose to present $\lambda_{0}=200 \mu \mathrm{m}$ but the other cases are similar. In Fig. 5 (right) we compare the analytical gain factor $(5)^{(2)}\left(k_{0}\right)$ defined by $(120)$ which we compute by Eq. (38) in [12] [the latter is an approximation

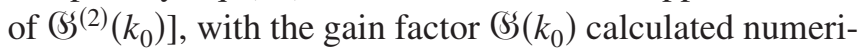
cally with our solver (red squares).

The analytical gain factor $(5)^{(2)}\left(k_{0}\right)$, which is derived in Sec. III C, is based on the coasting beam form (100) of $a_{0}$, i.e., based on the choice (131) of $\mu$ where, in the terminology of Sec. III,

$$
a_{C B}\left(p_{z}, x, p_{x}\right)=\rho_{c}\left(p_{z}\right) \rho_{t}\left(x, p_{x}\right) .
$$

The analytical gain factor $\left(5^{(2)}\left(k_{0}\right)\right.$ takes into account only CSR effects whereas our Vlasov-Maxwell approach automatically includes the effects of CSR and space charge in (3) $\left(k_{0}\right)$. Because our approach is much more detailed than the analytic approach we did not expect detailed agreement. Nonetheless, there does seem to be rough agreement in the $100-450 \mu \mathrm{m}$ range.

In the remaining parts of this section we deal with the numerical results of the MCP solver, i.e., with the choice (130) of $\mu$ and the gain factor $\mathbb{S}\left(k_{0}\right)$. The gain factor $\mathscr{S}\left(k_{0}\right)$ without self-fields is just the $\tilde{\rho}_{c}$ factor in (136), i.e., 

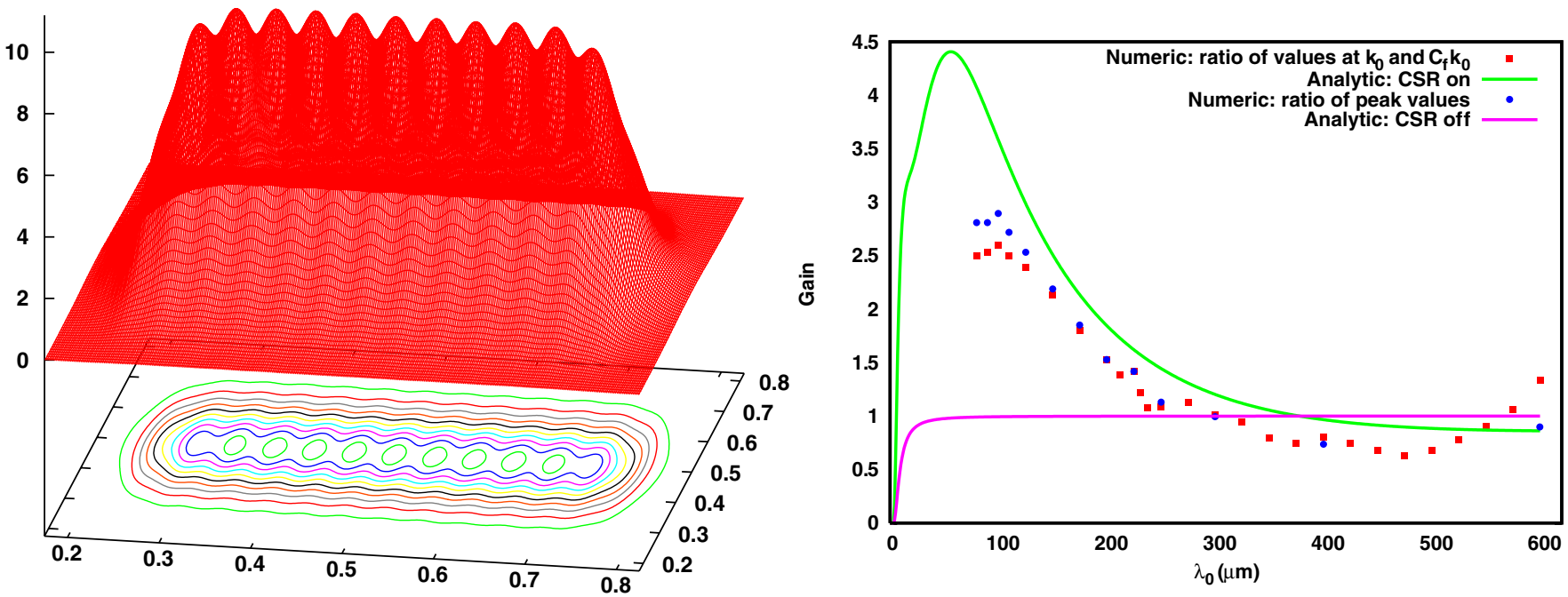

FIG. 5. (Color) Left: initial spatial density in grid coordinates $\left(x_{1}, x_{2}\right) \in[0,1] \times[0,1]$ for an initial modulation $\lambda_{0}=200 \mu \mathrm{m}$. Right: analytical gain factor ${ }^{(5)}\left(k_{0}\right)$ computed with analytical formula in [12] (curves) and numerical gain from our MCP solver (red squares and blue dots). The numerical gain is calculated as the ratio $\left(S\left(k_{0}\right)\right.$ of the values of the Fourier spectra at $k_{0}$ and $k_{0} C_{f}$ (red squares) and as the ratio of the peak values of the Fourier spectra (blue dots).

$$
\text { (S) } \begin{aligned}
\left(k_{0}\right) & =\left|\frac{\tilde{\rho}\left(k_{0} C_{f}, s_{f}\right)}{\tilde{\rho}\left(k_{0}, 0\right)}\right|=\left|\tilde{\rho}_{c}\left(k_{0} C_{f} R_{f}\right)\right| \\
& =\exp \left[-\left(2 \pi C_{f} R_{f} \sigma_{u}\right)^{2} / 2 \lambda_{0}^{2}\right],
\end{aligned}
$$

which is shown in Fig. 5 (right). For our parameter values, $\left(R_{f} \sigma_{u}\right)^{2} \approx 0.12 \times 10^{-6} \mathrm{~mm}^{2}$, thus the gain factor $\mathbb{S}\left(k_{0}\right)$ is essentially 1 except for an exponential fall off for $\lambda_{0}$ near zero.

To gain some understanding of Fig. 5 (right) we discuss the Fourier spectrum $|\tilde{\rho}(\cdot, s)|$ in some detail. Since $\rho$ is real the spectrum is an even function of $k$ and we only present it for positive $k$.

In Fig. 6 (left) we show the Fourier spectrum of $\rho$ at $s=$ 0 for $A=0$, i.e., $|\tilde{\mu}|$. As $\mu$ is close to $(\alpha / 2 a) I_{(-a, a)}(z)$ the Fourier spectrum $|\tilde{\mu}|$ is similar to $\alpha|\operatorname{sinc}(k a)|$. In Fig. 6 (right) we show the Fourier spectrum with self-fields at $s=$ $s_{f}$ without the initial modulation. Without self-fields we would have $\left|\tilde{\rho}\left(k, s_{f}\right)\right|=\exp \left[-\left(k R_{f} \sigma_{u}\right)^{2} / 2\right]\left|\tilde{\rho}\left(k / C_{f}, 0\right)\right|$. For our parameters and the range of $k$ in the figure, the exponential factor is 0.999 and so the $k$ axis is simply scaled by $C_{f}$. This is roughly what we see in Fig. 6 (right) which contains the self-fields. The first peak is at $3.763 \mathrm{~mm}^{-1}$ for $s=0$ and $13.125 \mathrm{~mm}^{-1}$ for $s=s_{f}$. The ratio is 3.488 as compared to 3.545 in the noncollective case. There is a visible decrease in the amplitude of the peak near $k=15 \mathrm{~mm}^{-1}$ and this must also be due to the collective effect.

Fourier spectra for $\lambda_{0}=300 \mu \mathrm{m}$ are shown in Fig. 7. In Fig. 7 (left) we show results for $s=0$ and the flattop and perturbed flattop. We note that the two spectra are essentially the same until $k \approx 16 \mathrm{~mm}^{-1}$. After this the flattop spectrum goes rapidly to zero whereas the perturbed spec-
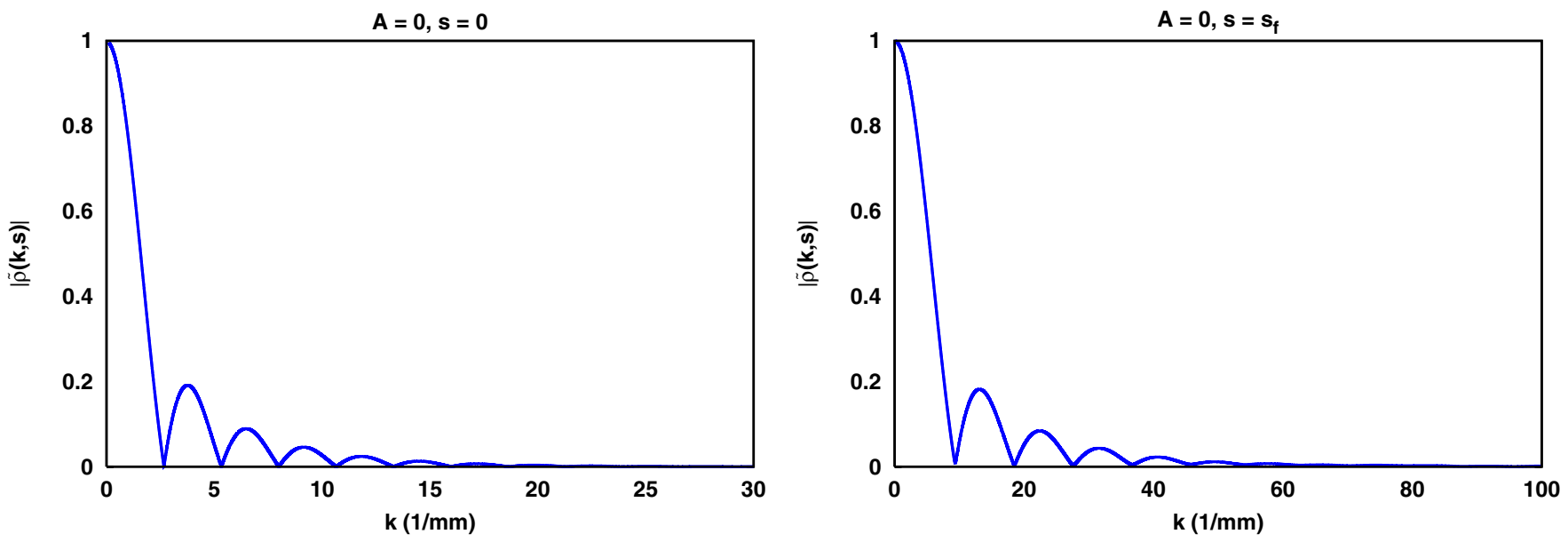

FIG. 6. (Color) Fourier spectra without perturbation $(\varepsilon=0)$. Left: at $s=0$; right: at $s=s_{f}$ with self-fields. 

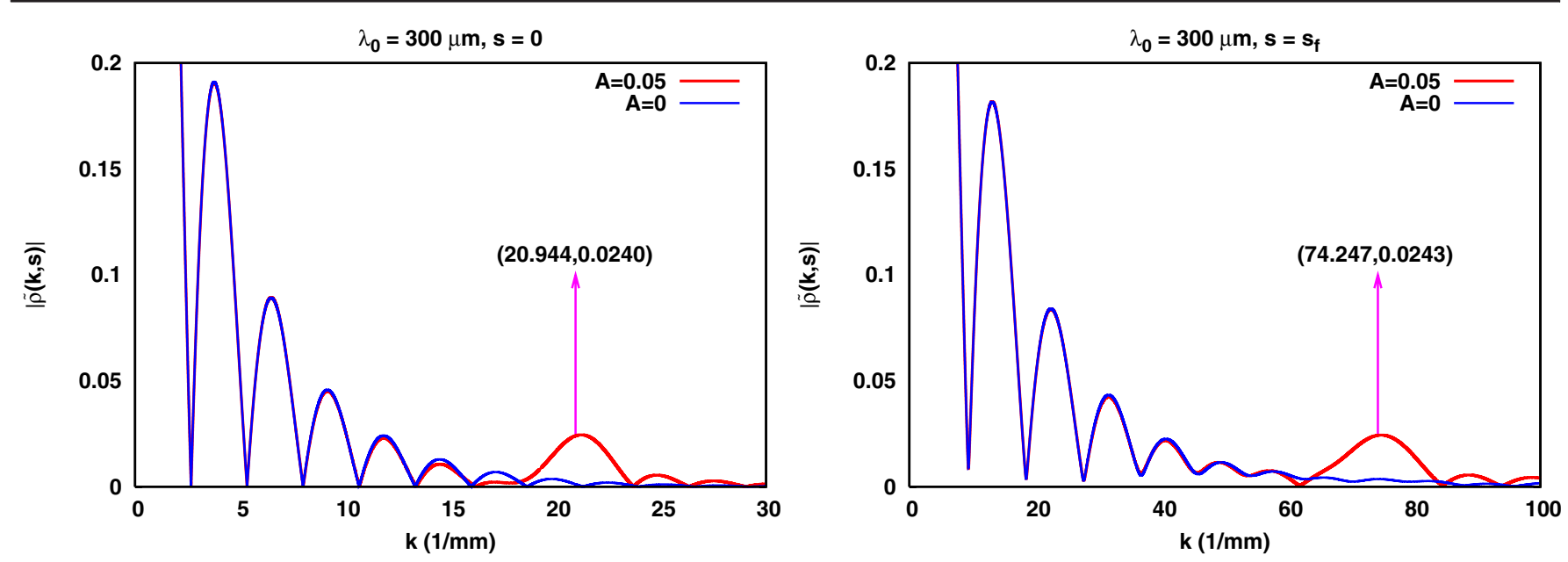

FIG. 7. (Color) Fourier spectra for $\lambda_{0}=300 \mu \mathrm{m}$ with and without perturbation at $s=0$ (left) and $s=s_{f}$ (right). The arrows indicate the points $\left\{k_{0} C(s), \tilde{\rho}\left[k_{0} C(s), s\right]\right\}$ at $s=0$ and $s=s_{f}$.

trum has a substantial contribution in a neighborhood of the perturbed wave number $k_{0}=20.94 \mathrm{~mm}^{-1}$. We note however that the peak is at a slightly larger $k$ value. We see a similar structure for the evolved spectra at $s=s_{f}$ in Fig. 7 (right), which is due to the fact that without selffields there is the simple scaling of (136) as we discussed in the context of Fig. 6. The effect of the collective force is seen in the diminution of the peak values of the first peak and a slight increase in the peak values near the arrows. The two Fourier spectra in Fig. 7 (right) are the same until $k \approx 60 \mathrm{~mm}^{-1}$. After this the flattop spectrum is nearly zero and the perturbed spectrum has a substantial contribution in a neighborhood of the perturbed but amplified wave number $k_{0} C_{f}=74.2 \mathrm{~mm}^{-1}$. The amplified wave number is slightly to the left of the peak. This raises the issue of how the gain should be defined from the Fourier spectrum. One possibility would be to take the ratio of the peak values. Figure 5 (right) shows the ratio of the peak values (blue dots) as well as the gain factor $\left(\$ s\left(k_{0}\right)\right.$, i.e., the

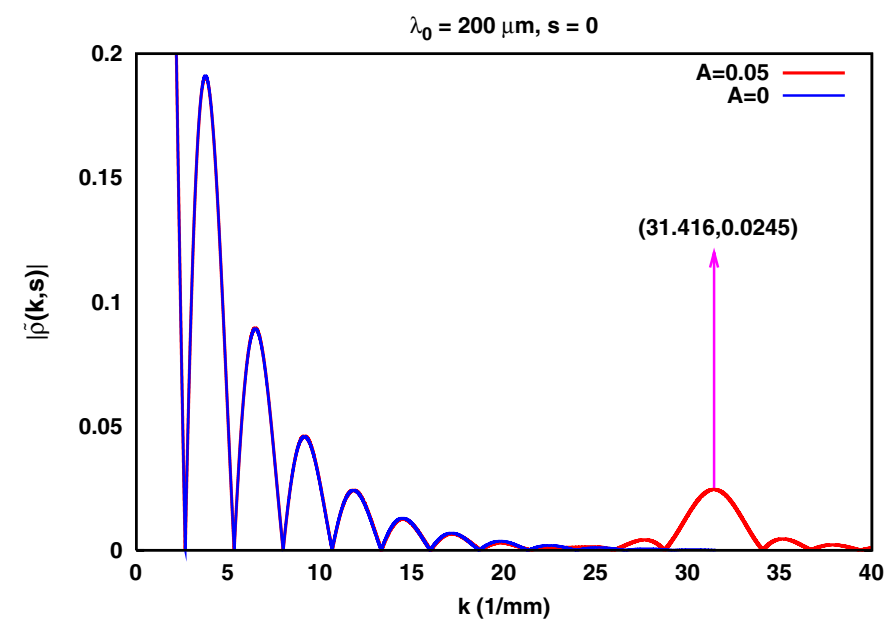

ratio of the values at $k_{0}$ and $k_{0} C_{f}$. Perhaps a better approach would be to consider some integral of $\tilde{\rho}(\cdot, s)$.

In Fig. 8 we show the same results for $\lambda_{0}=200 \mu \mathrm{m}$. Here the behavior is quite similar to the $\lambda_{0}=300 \mu \mathrm{m}$ case. However, there is less interference between the unperturbed and perturbed Fourier spectra causing the arrows to be closer to the peaks in the Fourier spectra. The collective effect is much increased over Fig. 7. There is a significant decrease in the peak values associated with the flattop and a significant increase in the peak values due to the perturbation.

The Fourier spectra at $s=0$ and $s=s_{f}$ for $\lambda_{0}=$ $100 \mu \mathrm{m}$ are shown in Fig. 9 (left) and, as in the $200 \mu \mathrm{m}$ case, there is no visible interference between the flattop and the perturbation. However, in contrast to the $200 \mu \mathrm{m}$ case, there is considerable enhancement and structure at the end of the chicane in the neighborhood of $k_{0} C_{f}=$ $222.741 \mathrm{~mm}^{-1}$. Defining the gain as the ratio of the values at the arrows appears to be somewhat arbitrary in two

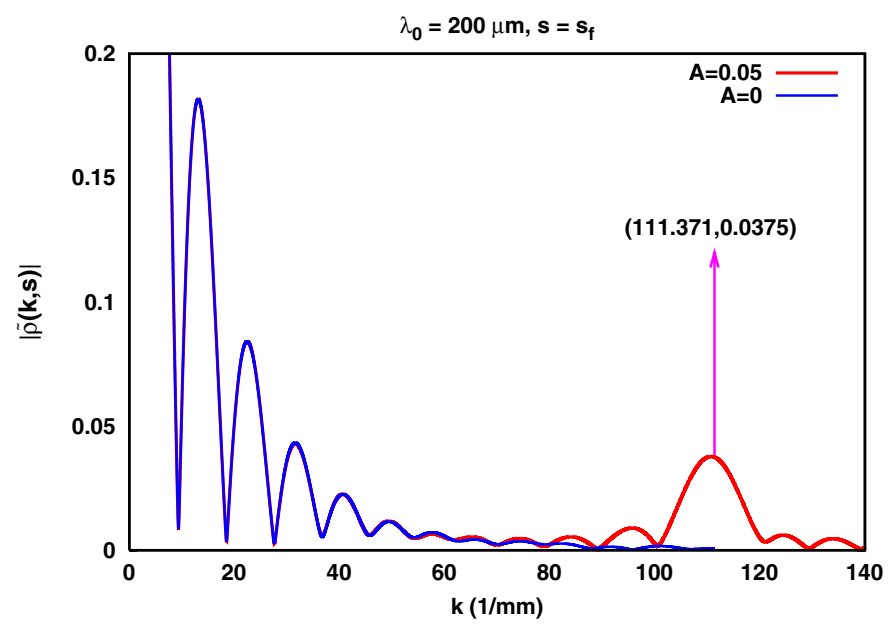

FIG. 8. (Color) Fourier spectra for $\lambda_{0}=200 \mu \mathrm{m}$ with and without perturbation at $s=0$ (left) and $s=s_{f}$ (right). Arrows as in Fig. 7. 

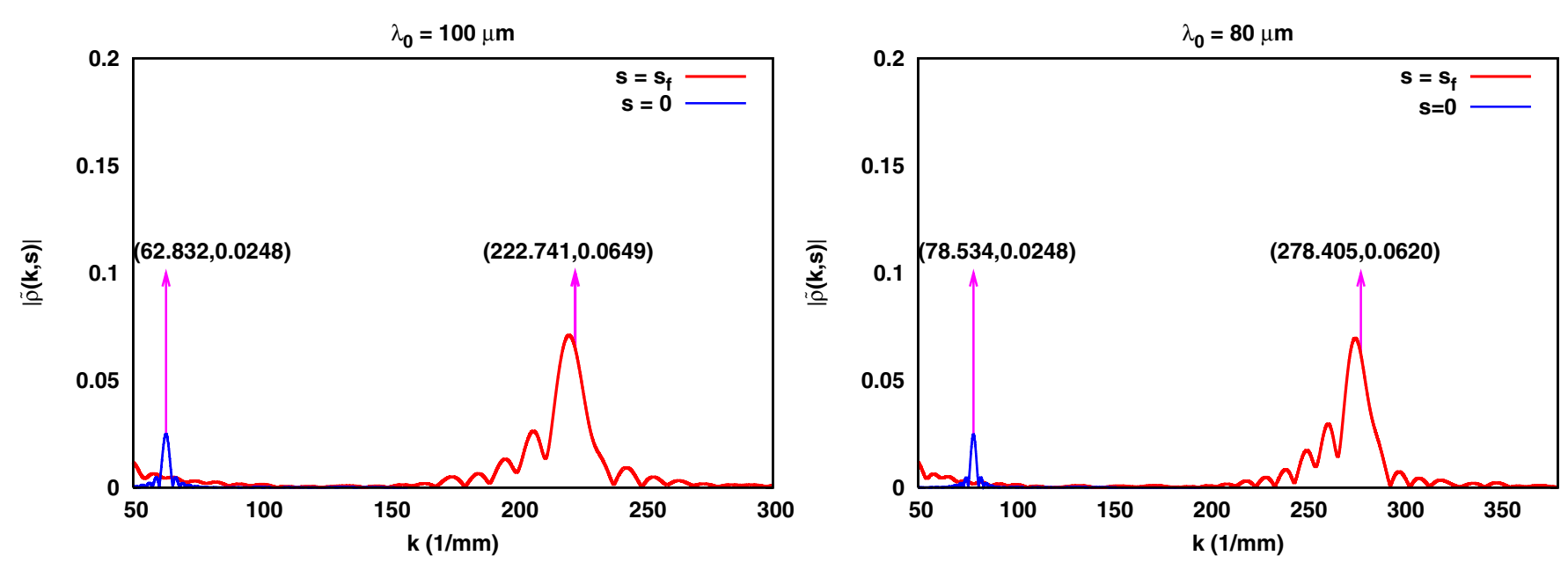

FIG. 9. (Color) Fourier spectra at $s=0$ and $s=s_{f}$ for $\lambda_{0}=100 \mu \mathrm{m}$ (left) and $\lambda_{0}=80 \mu \mathrm{m}$ (right). Arrows as in Fig. 7.

ways. One, the arrow at $s=s_{f}$ is to the right of the peak and two there is considerable structure between 200 and $250 \mathrm{~mm}^{-1}$.

At $80 \mu \mathrm{m}$ it appears that our calculation shows a decrease in the gain as indicated by the red squares and blue dots of Fig. 5 (right). However, this may be misleading as a comparison of the Fourier spectra in Fig. 9 (right) and Fig. 9 (left) does not clearly indicate a decrease in "gain" in going from $\lambda_{0}=100 \mu \mathrm{m}$ to $\lambda_{0}=80 \mu \mathrm{m}$. This further illustrates the need for a better definition of gain.

We are studying the Fourier spectra for $\lambda_{0}<80 \mu \mathrm{m}$ and preliminary results indicate a decrease in the gain and deviations from the analytical gain $\mathbb{S S}^{(2)}\left(k_{0}\right)$. The source of discrepancy might be due to nonlinearity, or to the different modeling of the collective force.

Currently we are running on Encanto at the New Mexico Computing Applications Center and on Franklin at the National Energy Research Scientific Computing Center (NERSC). Our run at $80 \mu \mathrm{m}$ takes $\approx 5$ hours using 800 processors. This along with memory considerations currently limits the minimum value of $\lambda_{0}$ we can study.

We have also considered values of $\lambda_{0}$ greater than $300 \mu \mathrm{m}$ as seen in Fig. 5 (right). The $\lambda_{0}=400 \mu \mathrm{m}$ case is shown in Fig. 10. Here we begin to see an overlap between the perturbed and unperturbed Fourier spectra and the arrows are quite a bit to the left of the peak values. The situation is even more severe at $600 \mu \mathrm{m}$, as can be seen in Fig. 11. At $400 \mu \mathrm{m}$ the red and blue markers in Fig. 5 (right) are very close and at $600 \mu \mathrm{m}$ the blue marker is almost on the analytical curve, but these are likely fortuitous results.

Recall now the coasting beam assumption that $\rho_{0}(z, s)$ is approximately independent of $z$ so that $\tilde{\rho}_{0}(k, s)$ is approximately zero for $k \neq 0$. What we see in Fig. 10 is that the Fourier spectra of $\rho_{0}$ and $\rho$ have significant overlap in the region where $k$ is near $k_{0}$ and $k_{0} C_{f}$. This signals the breakdown of the coasting beam assumption. The overlap is caused by the fact that there are only a small number of oscillations on the perturbed flattop. For $\lambda_{0}=400 \mu \mathrm{m}$
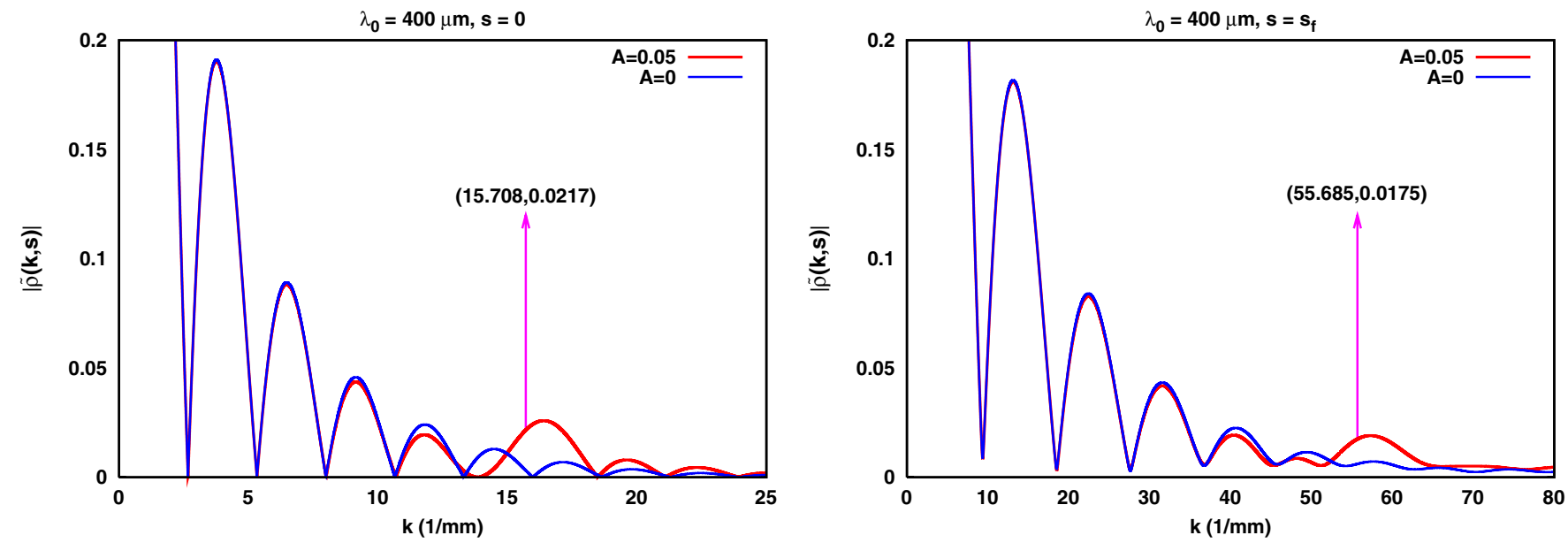

FIG. 10. (Color) Fourier spectra for $\lambda_{0}=400 \mu \mathrm{m}$ with and without perturbation at $s=0$ (left) and $s=s_{f}$ (right). Arrows as in Fig. 7 . 

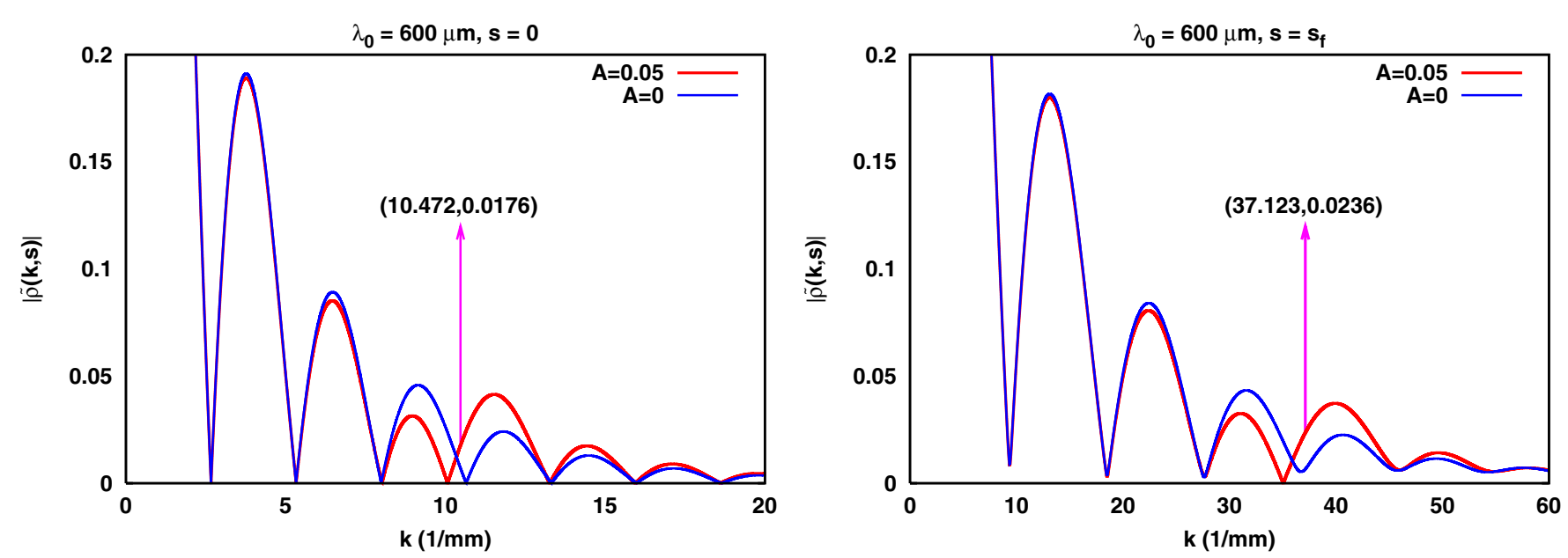

FIG. 11. (Color) Fourier spectra for $\lambda_{0}=600 \mu \mathrm{m}$ with and without perturbation at $s=0$ (left) and $s=s_{f}$ (right). Arrows as in Fig. 7 .

there are roughly four oscillations as can be deduced from the $\lambda_{0}=200 \mu \mathrm{m}$ case of Fig. 13.

The effect of the modulation on the average longitudinal force (mean power), $F_{z 1}$ of (27), and on the emittance $\epsilon_{x}=$ $\gamma_{r}\left(\sigma_{x}^{2} \sigma_{p x}^{2}-\sigma_{x p}^{2}\right)^{1 / 2}$ is very small for all values of $\lambda_{0}$. This is shown for $\lambda_{0}=100 \mu \mathrm{m}$ in Fig. 12. Notice that the emittance at $s_{f}$ is 1.5 times the initial one. The large values of the emittance away from the ends of the chicane is due to dispersion while the increase at the end of the chicane is totally due to the self-fields. There is no visible effect of the modulation even in this case of $\lambda_{0}=100 \mu \mathrm{m}$ where the modulation has the largest effect on $\tilde{\rho}\left(\cdot, s_{f}\right)$.

We have focused on the Fourier spectrum $|\tilde{\rho}(\cdot, s)|$ for $s=0$ and $s=s_{f}$ because the gain factor $\mathbb{S}\left(k_{0}\right)$ is defined in terms of this quantity. But it is also interesting to see the effect of the modulation on the longitudinal spatial density, $\rho(\cdot, s)$, itself.

In Figs. 13 and 14 we show results for the longitudinal spatial density $\rho$. The initial modulation width (min to $\max )$ is $2 A \mu(z=0)=42.4 \mathrm{~m}^{-1}$ thus the modulation width at $s=s_{f}$, without self-fields, given by (135) is $C_{f} 2 A \mu(z=0)=150.2 \mathrm{~m}^{-1}$.

In Fig. 13 (left) we show $\rho(\cdot, s)$ for $s=0$ and $s=s_{f}$ for $\lambda_{0}=200 \mu \mathrm{m}$ with no self-fields. The compression is obvious and it is important to note that the modulation width is enhanced by the compression factor $C_{f}$. So there is an enhancement without self-fields. As mentioned before, (135) gives the excellent approximation $\rho\left(z, s_{f}\right)=$ $C_{f} \rho\left(C_{f} z, 0\right)$ and a plot of this cannot be distinguished from that in Fig. 13 (left).
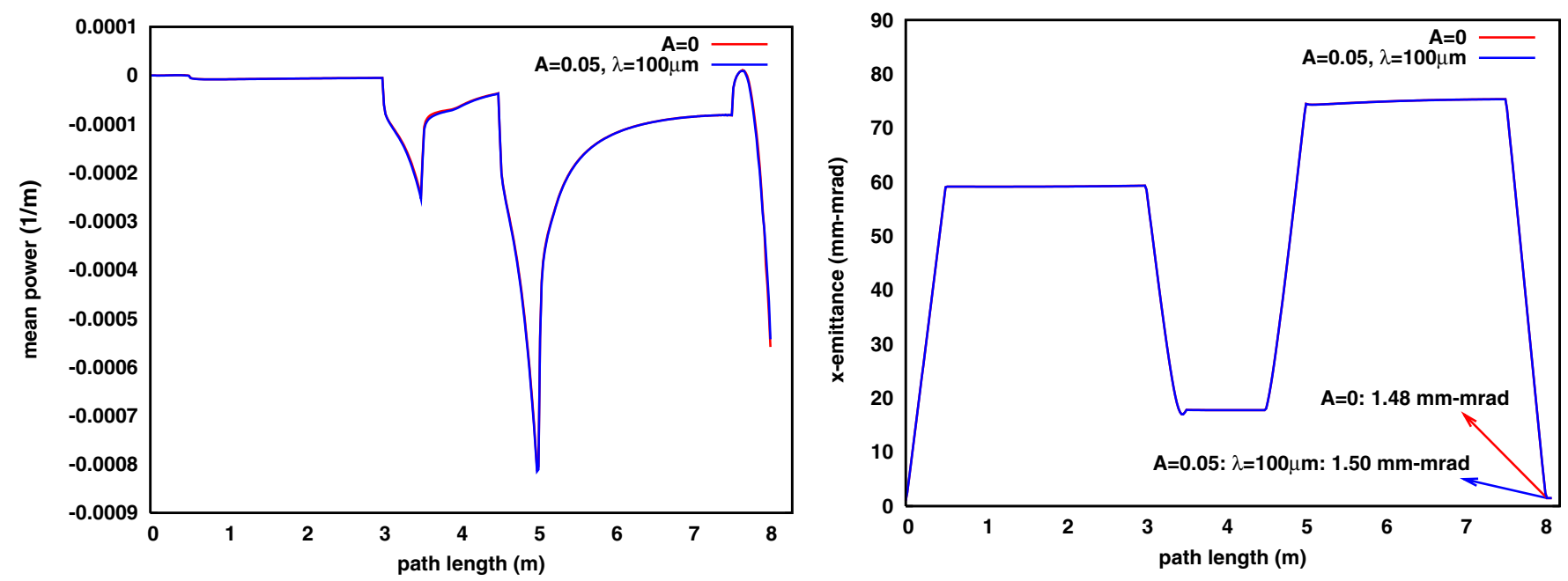

FIG. 12. (Color) Left: mean power vs $s$ with no initial modulation $(A=0)$ (red curve) and with $A=0.05, \lambda_{0}=100 \mu \mathrm{m}$ (blue curve). Right: same as left frame for $x$ emittance. Notice that the effect of the initial modulation on the mean power and emittance is negligible. 

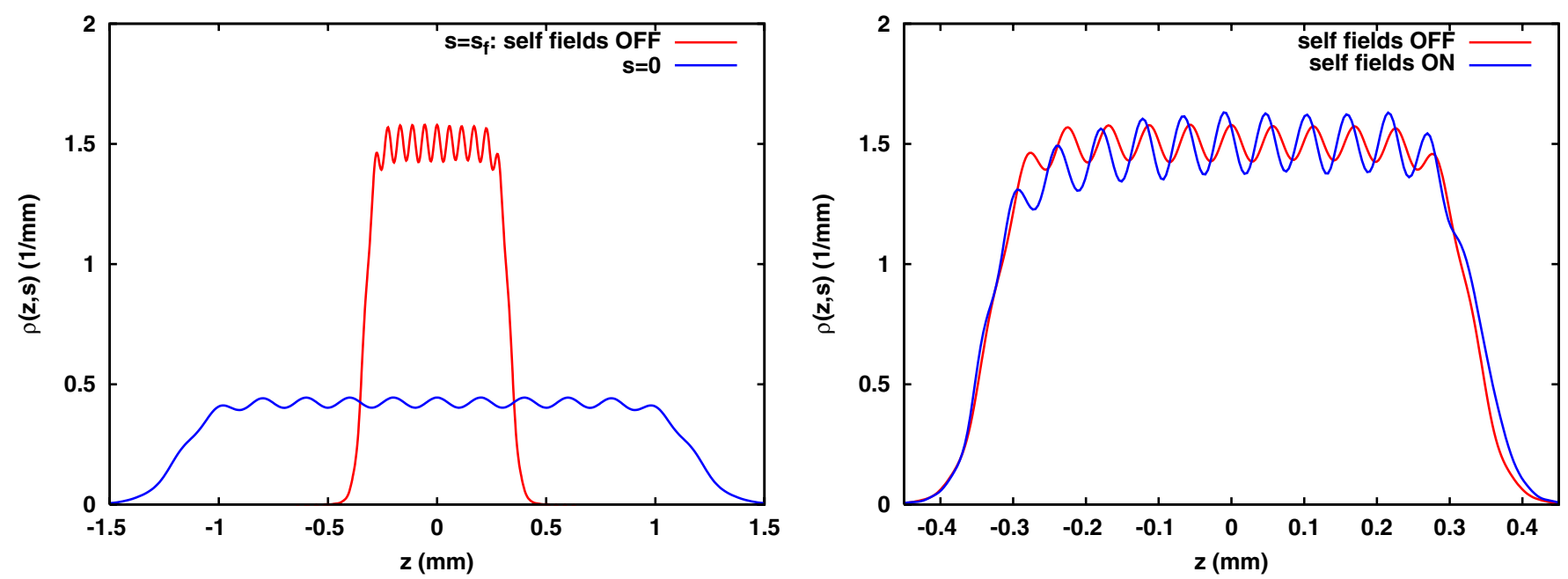

FIG. 13. (Color) Longitudinal spatial density for $\lambda_{0}=200 \mu \mathrm{m}$. Left: $s=0$ and $s=s_{f}$ without self-fields. Right: $s=s_{f}$ with and without self-fields.

In Fig. 13 (right) we show $\rho\left(\cdot, s_{f}\right)$ for $\lambda_{0}=200 \mu \mathrm{m}$ both with and without the collective force. One can see the enhancement of the modulation width with the collective force, over and above that without the collective force. The modulation width is now approximately $200 \mathrm{~m}^{-1}$. Also an asymmetry due to the collective force has appeared and the fraction of the bunch for $z<0$ has decreased.

In Fig. 14 (left) the setup is the same as in Fig. 13 (right) except $\lambda_{0}$ is now $100 \mu \mathrm{m}$. This is the case of maximum gain in Fig. 5 (right). The results are similar to the $200 \mu \mathrm{m}$ case except the modulation width is now approximately $500 \mathrm{~m}^{-1}$, a considerable enhancement over both the $200 \mu \mathrm{m}$ case as well as the case without self-fields. This is to be expected from Fig. 5 (right).

Figure 14 (right) repeats the setup of Figs. 13 (right) and 14 (left) for $\lambda_{0}=80 \mu \mathrm{m}$. Here we see a slight increase in the modulation width to approximately $650 \mathrm{~m}^{-1}$. Thus even though there is a decrease in the gain at $\lambda_{0}=$ $80 \mu \mathrm{m}$ in Fig. 5 (right), this is not seen in the modulation width nor in the associated Fourier spectrum.

An important part of our current calculation is that we have found an $s$-independent grid for the spatial density that allows us to minimize the number of grid points as discussed in Sec. II C. Recall that this grid is based on the $\left(x_{1}, x_{2}\right)$ coordinates introduced in the context of (38) and in these coordinates the spatial density is stationary for $\sigma_{u}=$ $\sigma_{p_{x 0}}=0$ and no self-fields.

In Fig. 15 we present the evolution of the spatial density for $\lambda_{0}=200 \mu \mathrm{m}$ without self-fields. The density is shown in Fig. 5 (left) at $s=0$ and in Fig. 15 for $s=0.25 s_{f}, s=$ $0.5 s_{f}, s=0.75 s_{f}$, and $s=s_{f}$. The grid coordinates $\left(x_{1}, x_{2}\right)$ which were introduced in Sec. IIC are used. There is no visual change in the spatial density nor in the contour plots showing that the effect of nonzero $\sigma_{u}$ and
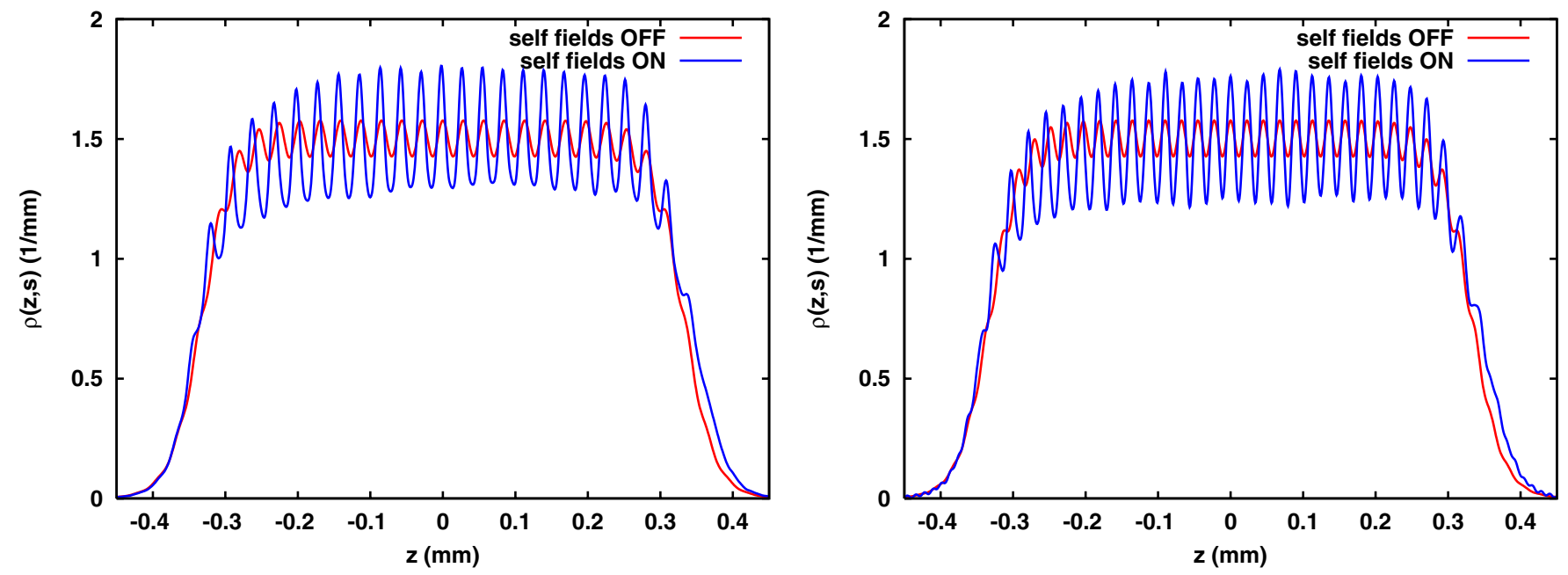

FIG. 14. (Color) Longitudinal spatial density at $s=s_{f}$ with and without self-fields for $\lambda_{0}=100 \mu \mathrm{m}$ (left) and $\lambda_{0}=80 \mu \mathrm{m}$ (right). 

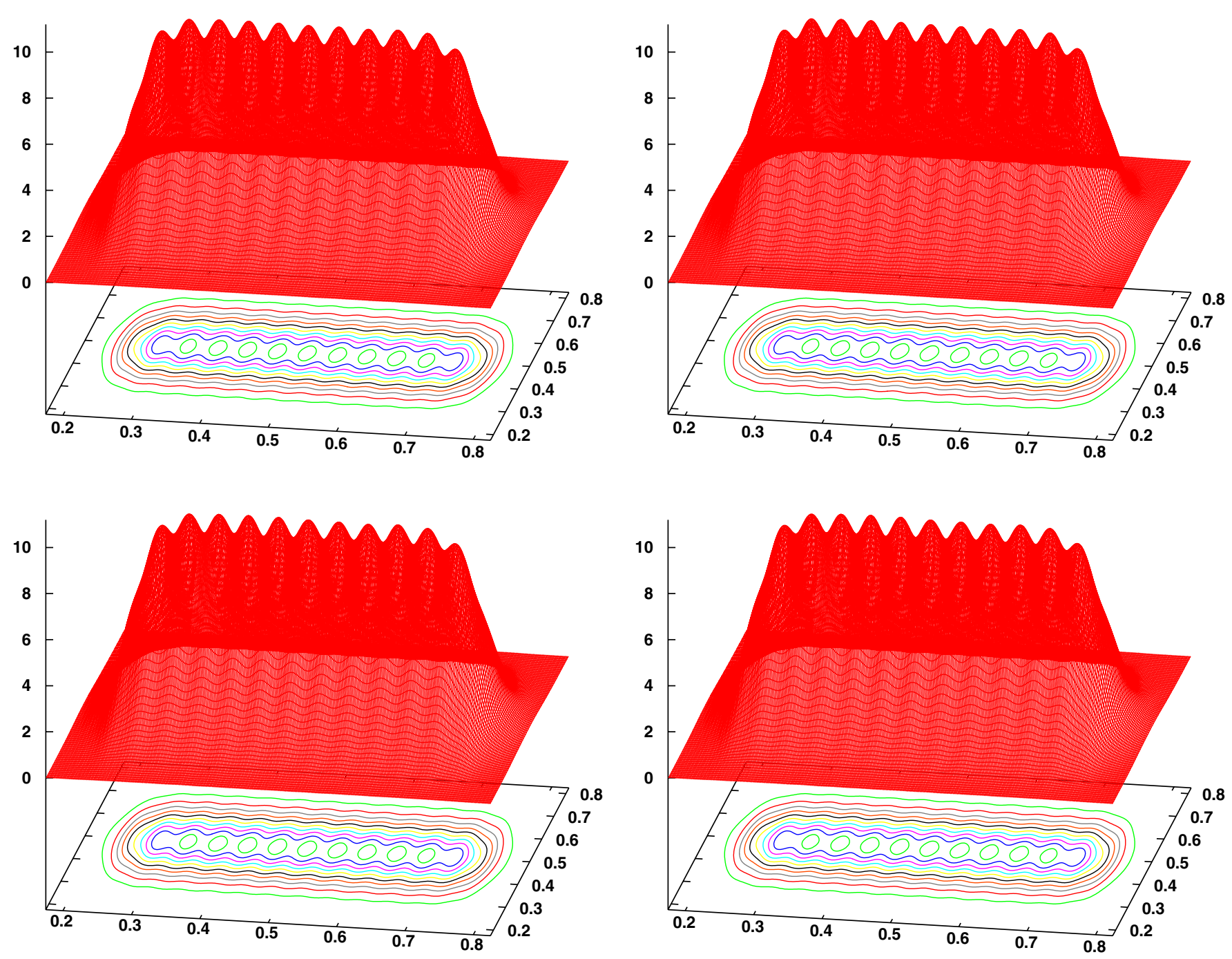

FIG. 15. (Color) Spatial density in grid coordinates $\left(x_{1}, x_{2}\right)$ with no self-fields for $\lambda_{0}=200 \mu \mathrm{m}$ at $s=0.25 s_{f}$ (top left), $s=0.5 s_{f}$ (top right), $s=0.75 s_{f}$ (bottom left), and $s=s_{f}$ (bottom right).

$\sigma_{p_{x 0}}$ is not important. (Here $\sigma_{p_{x 0}}=1.5 \times 10^{-5}$ and recall that $\sigma_{u}=8.6 \times 10^{-6}$ ) This means that, even though there are large changes in the density in beam frame coordinates as it evolves through the chicane [recall Fig. 12 (right)], the $\left(x_{1}, x_{2}\right)$ coordinates allow us to eliminate that effect in the calculations.

Next we present density plots with self-fields. In Fig. 16 we show the spatial density in grid coordinates $\left(x_{1}, x_{2}\right)$ for $\lambda_{0}=200 \mu \mathrm{m}$ (left) and $\lambda_{0}=100 \mu \mathrm{m}$ (right) at $s=s_{f}$. Here we simply reiterate that we are able to calculate accurately this $2 \mathrm{D}$ spatial density, the basic quantity in our 2D Ansatz.

In Fig. 17 we show the longitudinal force $F_{z 1}$, proportional to $\mathbf{E}_{L} \cdot \mathbf{t}$, at $s=s_{f}$ for $\lambda_{0}=200 \mu \mathrm{m}$ (left) and $100 \mu \mathrm{m}$ (right). Notice that the maximum intensity of $F_{z 1}$ increases as $\lambda_{0}$ decreases, a behavior similar to the oscillation amplitude of the spatial densities in Figs. 13 and 14, and in Fig. 16.
In a convergence study we compared simulation results for $\lambda_{0}=200 \mu \mathrm{m}$ at $s=s_{f}$ for two sets of parameters $\mathcal{N}_{1}=60 \times 10^{6}, \quad I_{1}=60, \quad J_{1}=30 \quad$ and $\quad \mathcal{N}_{2}=$ $120 \times 10^{6}, I_{2}=90, J_{2}=50$. The comparison for the longitudinal spatial density is shown in Fig. 18. We have also compared 2D plots of the spatial density at the two levels of resolution. These are indistinguishable by eye and so we do not show them. The gain, energy loss, and emittance for the set of parameters $\left(\mathcal{N}_{1}, I_{1}, J_{1}\right)$ are 1.5291, -0.0007936 , and 1.4498 ( $\mathrm{mm}$ mrad), respectively, while the same quantities for the set of parameters $\left(\mathcal{N}_{2}, I_{2}, J_{2}\right)$ are $1.5282,-0.0007931$, and $1.4501(\mathrm{~mm} \mathrm{mrad})$, respectively.

Our MCP solver, based on a Monte Carlo particle method to solve the Vlasov equation and a Green function method to solve the Maxwell equations, demonstrated high efficiency and scalability. Its high performance has been tested on several parallel clusters. The number of particles 

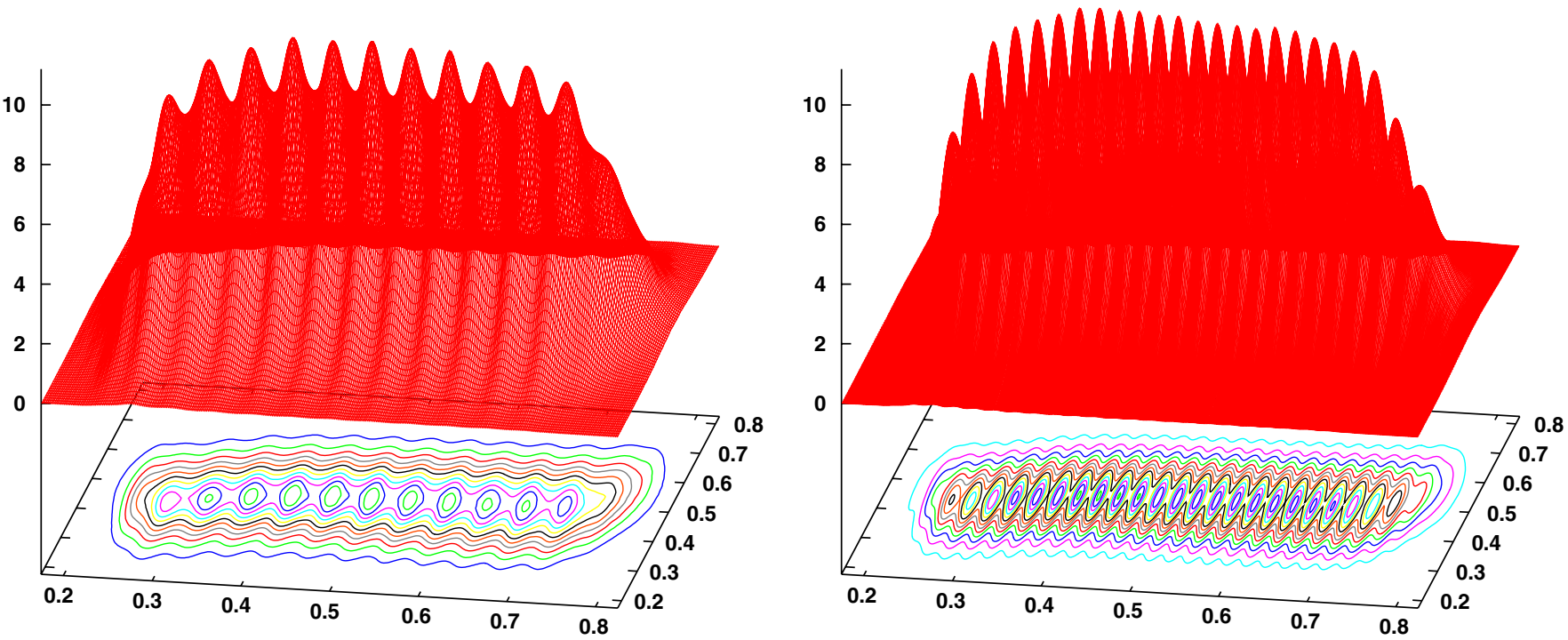

FIG. 16. (Color) Spatial density in grid coordinates $\left(x_{1}, x_{2}\right)$ at $s=s_{f}$ for $\lambda_{0}=200 \mu \mathrm{m}$ (left) and $\lambda_{0}=100 \mu \mathrm{m}$ (right).

$\mathcal{N}$ used in the simulations varies from few tens to few hundreds of millions and the number of Fourier coefficients $(I, J)$ used in the estimation of the 2D charge/current density runs from $(30,30)$ to $(120,50)$. The number of grid points $\left(N_{x 1}, N_{x 2}\right)$ in grid coordinates $\left(x_{1}, x_{2}\right)$ is fixed by $(I, J)$ as follows. We determine $N_{x 1}$ and $N_{x 2}$ in order to have 15-20 grid points per wavelength of the basis functions $\sqrt{2} \cos \left(I \pi x_{1}\right)$ and $\sqrt{2} \cos \left(J \pi x_{2}\right)$, respectively. For a particular simulation the grid extent is $6 \sigma_{z}=4.6 \mathrm{~mm}$ thus for a given modulation $\lambda_{0}$, for example, for $\lambda_{0}=100 \mu \mathrm{m}$, the value of $J$ must be bigger than 92 . To determine $\mathcal{N}$ we define an error $\epsilon$ as the square of the $L^{2}$ norm of $\rho_{\text {est }}-\rho_{\text {an }}$, where $\rho_{\text {est }}$ is the estimated spatial density and $\rho_{\text {an }}$ is the spatial density given analytically. We choose $\mathcal{N}$ in order to have $\epsilon \leq 10^{-5}$.

The study of the gain factor at short wavelengths is computationally expensive. Moreover, the increased length of the 3D arrays needed to store the history of the charge/ current densities leads to intensive memory usage. Studies are in progress to investigate wavelengths shorter than $\lambda_{0}=80 \mu \mathrm{m}$ and different amplitudes $A$.

An important prediction of the gain formula from [12], i.e., from the analytical gain factor $\mathbb{S S}^{(2)}\left(k_{0}\right)$, is that increasing the uncorrelated energy spread reduces the gain factor. This led to a proposal, the laser heater, to increase the uncorrelated energy spread within FEL tolerance in order
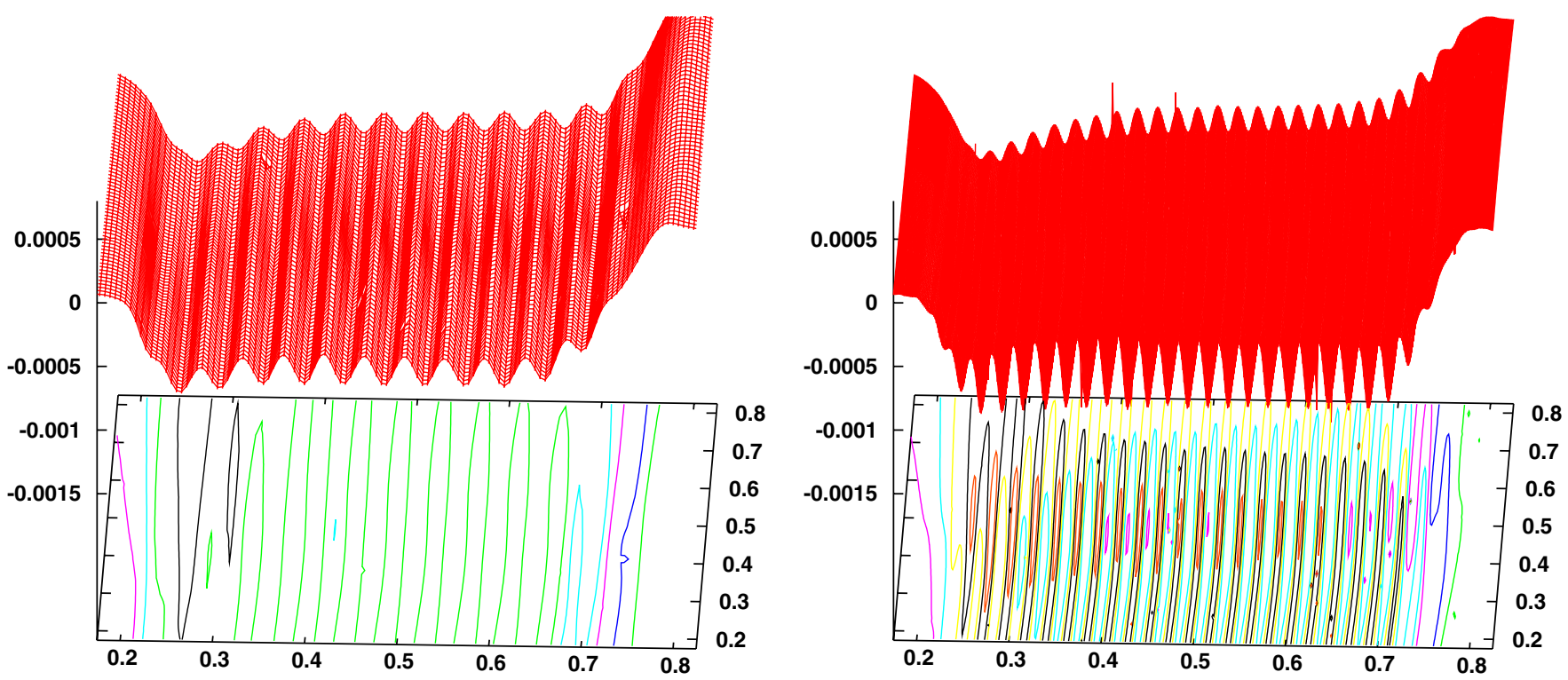

FIG. 17. (Color) Longitudinal force in grid coordinates $\left(x_{1}, x_{2}\right)$ at $s=s_{f}$ for $\lambda_{0}=200 \mu \mathrm{m}$ (left) and $\lambda_{0}=100 \mu \mathrm{m}$ (right). 


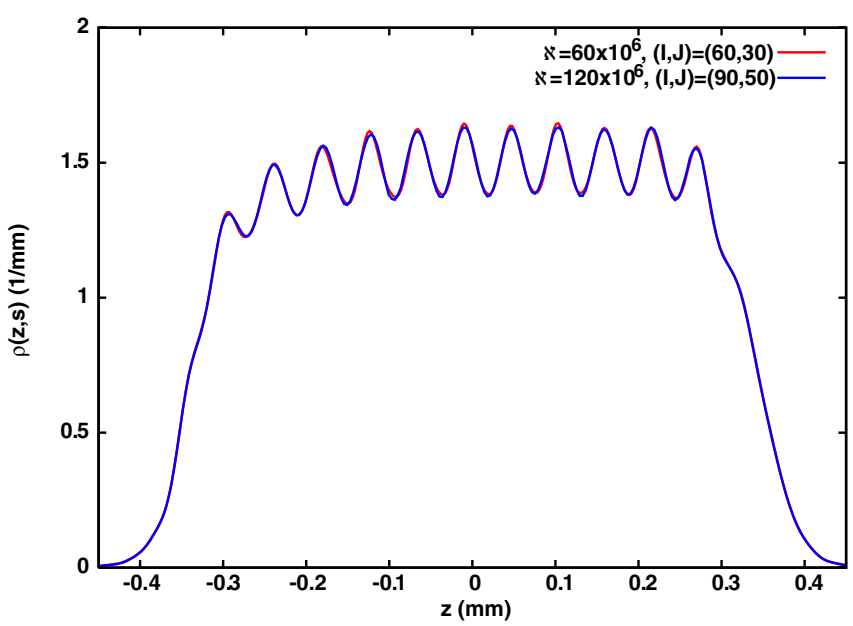

FIG. 18. (Color) Comparison of the longitudinal spatial density for $\lambda_{0}=200 \mu \mathrm{m}$ at $s=s_{f}$ for the set of parameters $\mathcal{N}_{1}=$ $60 \times 10^{6}, I_{1}=60, J_{1}=30$ and $\mathcal{N}_{2}=120 \times 10^{6}, I_{2}=90$, $J_{2}=50$.

to damp the microbunching instability without degrading the FEL performance. An analysis of this effect together with the complete study of the FERMI@Elettra benchmark bunch compressor system is in progress.

\section{CONCLUSIONS}

We have demonstrated a procedure with some new features for self-consistent simulation of coherent motion, with application to a bunch compressor. Although it is based on tracking an ensemble of particles, as in usual macroparticle or PIC codes, the method of smoothing the charge distribution is quite different, using a Fourier expansion with Monte Carlo determination of the expansion coefficients. The resulting smooth distribution is used in an accurate solution of the field equations by a Green function method. We hope that the resulting time evolution of the spatial density approximates that which would be obtained from a solution of the Vlasov-Maxwell system on the 4D phase space, but there is no direct check on accuracy of such an approximation. However, the evident lack of noise in the simulation is encouraging.

Using $10^{7}-10^{8}$ particles and an adequate number of Fourier modes, we are able to study amplification of initial density modulation down to a wavelength of $80 \mu \mathrm{m}$, in the example of the first chicane bunch compressor at FERMI@Elettra. We see clean amplification at the compressed value of the initial modulation wavelength, in accord with the prediction of the linear theory except at the smallest wavelengths. Even at $80 \mu \mathrm{m}$ the modulation has a negligible effect on the final emittance and energy loss, although the gain is large.

We anticipate improvements in the code regarding treatment of the spatial density, but at present the most costly part is the field calculation. We intend to review the choice of integration variables and the integration algorithms to see if the field evaluation can be speeded up. Parts of the integration, for large retarded times, may have been done more accurately than necessary.

We have reviewed the linearized Vlasov equation for single-pass systems. We first avoid assumptions used previously, for instance by linearizing about an arbitrary solution of the Vlasov equation rather than about a coasting beam solution with linear energy chirp. Still assuming a 1D description of the collective force, we find in general that the linear Vlasov equation can be stated as an integral equation in a $2 \mathrm{D}$ space for the Fourier transform of the longitudinal spatial density, $\tilde{\rho}(k, s)$. This applies to a bunched beam, and allows arbitrary chirp. For linearization about a coasting beam with linear energy chirp, we retrieve the equation previously known. Study of the general equation is feasible and of some interest, especially regarding nonlinear chirp.

\section{ACKNOWLEDGMENTS}

We gratefully acknowledge the help of T. Thomas at UNM HPC, and thank R. Ryne and P. Spentzouris for an account on NERSC. M. Venturini made valuable comments on the integral equation. This work has been partially supported by U.S. Department of Energy Contracts No. AC02-76SF00515 and No. DE-FG-99ER41104.

[1] M. Borland, Phys. Rev. ST Accel. Beams 11, 030701 (2008).

[2] M. Venturini, R. Warnock, and A. Zholents, Phys. Rev. ST Accel. Beams 10, 054403 (2007).

[3] M. Venturini, Phys. Rev. ST Accel. Beams 10, 104401 (2007).

[4] M. Venturini and A. Zholents, Nucl. Instrum. Methods Phys. Res., Sect. A 593, 53 (2008).

[5] M. Venturini, Phys. Rev. ST Accel. Beams 11, 034401 (2008).

[6] E. L. Saldin, E. A. Schneidmiller, and M. V. Yurkov, Nucl. Instrum. Methods Phys. Res., Sect. A 490, 1 (2002).

[7] G. Angelova, V. Ziemann, M. Dohlus, and Y. Kot, in Proceedings of the 11th European Particle Accelerator Conference, Genoa, 2008 (EPS-AG, Genoa, Italy, 2008).

[8] T. Shaftan and Z. Huang, Phys. Rev. ST Accel. Beams 7, 080702 (2004).

[9] Z. Huang, J. Wu, and T. Shaftan, SLAC Report No. SLACPUB-11597, 2005.

[10] Z. Huang, M. Borland, P. Emma, J. Wu, C. Limborg, G. Stupakov, and J. Welch, Phys. Rev. ST Accel. Beams 7, 074401 (2004).

[11] D. C. Sagan, G. H. Hoffstaetter, C. E. Mayes, and U. SaeUeng, Phys. Rev. ST Accel. Beams 12, 040703, (2009).

[12] H. Huang and K. Kim, Phys. Rev. ST Accel. Beams 5, 074401 (2002); 5, 129903 (2002).

[13] S. Heifets, G. Stupakov, and S. Krinsky, Phys. Rev. ST Accel. Beams 5, 064401 (2002); 5, 129902 (2002). 
[14] https://www.elettra.trieste.it/FERMI/index.php?n=Main. MicrobProgram.

[15] J. A. Ellison, G. Bassi, K. Heinemann, M. Venturini, and R. Warnock, in Proceedings of the 2007 Particle Accelerator Conference, Albuquerque, New Mexico, 2007 (IEEE, Albuquerque, New Mexico, 2007).

[16] G. Bassi, J. A. Ellison, K. Heinemann, and R. Warnock, in Proceedings of the 2007 Particle Accelerator Conference, Albuquerque, New Mexico, 2007 (Ref. [15]).

[17] H. Huang, M. Borland, P. Emma, and K. Kim, Nucl. Instrum. Methods Phys. Res., Sect. A 507, 318 (2003).

[18] L.C. Evans, Partial Differential Equations (American Mathematical Society, Providence, 1998).

[19] G. Bassi, J. A. Ellison, and R. Warnock (unpublished).

[20] R. Li, Phys. Rev. ST Accel. Beams 11, 024401 (2008).

[21] R. Warnock, J. A. Ellison, K. Heinemann, and G. Q. Zhang, in Proceedings of the 11th European Particle Accelerator Conference, Genoa, 2008 (Ref. [7]) (TUPP109).

[22] S. M. Ross, Simulation (Academic Press, San Diego, 1997).

[23] S. Efromovich, Nonparametric Curve Estimation: Methods, Theory, and Applications (Springer, Berlin, 1999).
[24] R. E. Caflisch, Acta Numerica 7, 1 (1998).

[25] H. Niederreiter, Random Number Generation and QuasiMonte Carlo Methods (SIAM, Philadelphia, 1992).

[26] S. Kunis and D. Potts, NFFT, Softwarepackage, C Subroutine Library http://www.tu-chemnitz.de/potts/nfft (2002-2005).

[27] B. Terzic, I. V. Pogolerov, and C. Bohn, Phys. Rev. ST Accel. Beams 10, 034201 (2007).

[28] B. W. Silverman, Density Estimation (Chapman and Hall, London, 1986).

[29] J. Fan and I. Gijbels, Local Polynomial Modeling and Its Applications (Chapman and Hall, London, 1996).

[30] G. E. Fasshauer, Meshfree Approximation Methods with MATLAB (World Scientific, Singapore, 2007).

[31] M.D. Buhmann, Radial Basis Functions, Theory and Implementations (Cambridge University Press, Cambridge, England, 2003).

[32] H. Wendland, Scattered Data Approximation (Cambridge University Press, Cambridge, England, 2005).

[33] M. Venturini (private communication).

[34] M. Abramowitz and I. A. Stegun, Handbook of Mathematical Functions (National Bureau of Standards, Washington, D.C., 1964), Sec. 10.4.32. 